\title{
Multi-Bifurcation Cascaded Bursting Oscillations and Mechanism in a Novel 3D Non-autonomous Circuit System with Parametric and External Excitation
}

mengjiao wang ( $\square$ wangmj@xtu.edu.cn )

Xiangtan University https://orcid.org/0000-0002-8457-1307

Jianhui Li

Xiangtan University

Xinan Zhang

University of Western Australia

Herbert Ho-Ching lu

University of Western Australia

Tyrone Fernando

University of Western Australia

Zhijun Li

Xiangtan University

Yicheng Zeng

Xiangtan University

\section{Research Article}

Keywords: Multi-timescale system , Bifurcation , Bursting oscillation , Fast-slow dynamics, Chaotic dynamics

Posted Date: March 19th, 2021

DOI: https://doi.org/10.21203/rs.3.rs-322891/v1

License: (c) (i) This work is licensed under a Creative Commons Attribution 4.0 International License.

Read Full License

Version of Record: A version of this preprint was published at Nonlinear Dynamics on August 4th, 2021.

See the published version at https://doi.org/10.1007/s11071-021-06763-y. 


\title{
Multi-Bifurcation Cascaded Bursting Oscillations and Mechanism in a Novel 3D Non-autonomous Circuit System with Parametric and External Excitation
}

\author{
Mengjiao Wang · Jianhui Li · Xinan Zhang · Herbert Ho-Ching Iu · Tyrone \\ Fernando · Zhijun Li · Yicheng Zeng
}

Received: date / Accepted: date

\begin{abstract}
In this paper, multi-timescale dynamics and the formation mechanism of a 3D non-autonomous system with two slowly varying periodic excitations are systematically investigated. Interestingly, the system shows novel multibifurcation cascaded bursting oscillations (MBCBOs) when the frequency of the two excitations is much lower than the mean frequency of the original system (MFOS). For instance, periodic, quasi-periodic and chaotic bursting oscillations induced by a variety of cascaded bifurcations are first observed, and the phenomenon of spiking transfer is also revealed. Besides, stability and local bifurcations of the system are comprehensively investigated to analyze the mechanism of the observed MBCBOs, in which bifurcation diagram, Lyapunov exponents, time series, phase portraits, and transformed phase diagrams are used. Finally, through a circuit simulation and hardware experiment, these complex dynamics phenomena are verified physically.
\end{abstract}

Keywords Multi-timescale system · Bifurcation · Bursting oscillation · Fast-slow dynamics $\cdot$ Chaotic dynamics

M. Wang $(\bowtie)$

School of Automation and Electronic Information, Xiangtan University, Xiangtan, 411105, P. R. China

School of Electrical, Electronic and Computer Engineering, University of Western Australia, Crawley, WA 6009, Australia

E-mail: wangmj@xtu.edu.cn

J. Li $\cdot$ Z. Li

School of Automation and Electronic Information, Xiangtan University, Xiangtan, 411105, P. R. China

X. Zhang · H.H.C. Iu · T. Fernando

School of Electrical, Electronic and Computer Engineering, University of Western Australia, Crawley, WA 6009, Australia

Y. Zeng

School of Physics and Optoelectric Engineering, Xiangtan University, Xiangtan, 411105, P. R. China

\section{Introduction}

Multi-timescale dynamics are ubiquitous in diverse research fields. For example, by regulating calcium and potassium activity, the biophysical model exhibits the spontaneous bursting behavior in the developing retina [1], complex mixedmode oscillatory patterns are revealed in a Belousov-Zhabtinsky reaction with fast-slow timescale [2], the coexisting fastscale and slow-scale bifurcation are observed in simple dc/dc converters [3] and the complicated bursting oscillations are generated in the hydroelectric power system with multiple time scales [4]. In 1963, Lorenz discovered the first chaotic system when studying the weather model [5]. Thereafter, various chaotic systems have been proposed by many scholars, especially Lorenz system family. As a type of classically chaotic system, Lorenz system family has been extensively studied and applied in the past decades. However, most of the work focused on the study of dynamic characteristics at the same timescale [6-8], and the multi-timescale dynamics of Lorenz system family is rarely reported. Therefore, "what kind of dynamic behavior can Lorenz system family with multi timescale and multi-frequency periodic excitation exhibit?" remains an open question that is worth studying further.

Bursting oscillations (BOs) caused by periodic switching of the spiking state (SP) and the quiescent state (QS) [9], as an interesting multi-timescale dynamics, has great potential for applications. For example, researchers in [10] used several types of BOs to drive electrodynamical systems. Authors in [11] demonstrated a specific type of artificial neuron with threshold-driven spiking and built a neural network for pattern recognition. Furthermore, it can also be applied to the study of stability and control design of pulsed power supply systems according to similar oscillation characteristics [12]. Therefore, the exploration of new patterns of BO can not only supplement the existing theory but also 


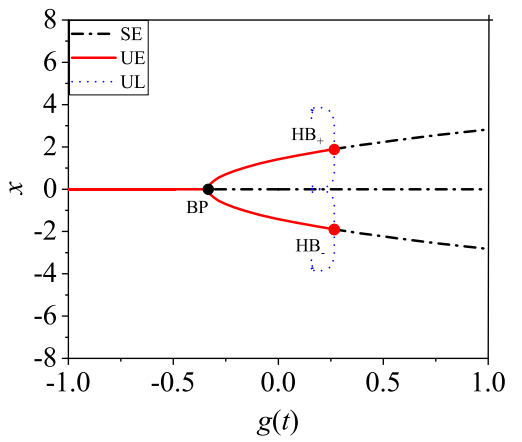

(a)

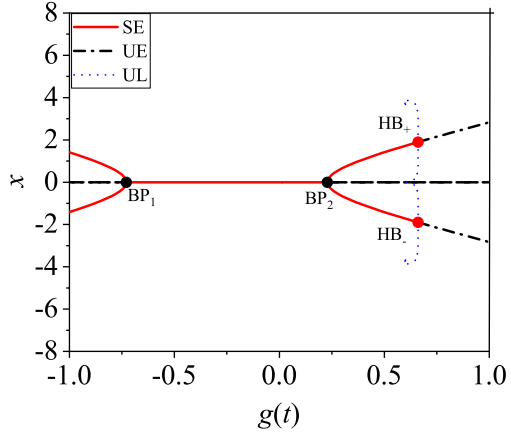

(b)

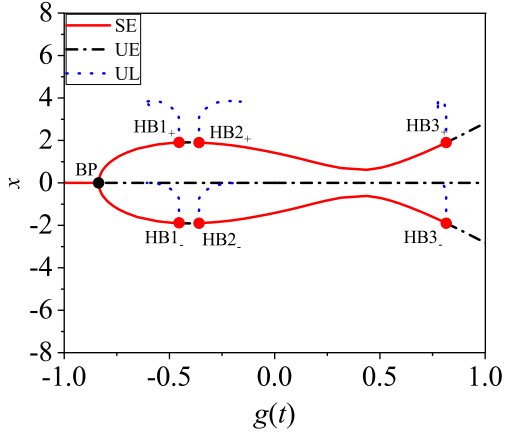

(c)

Fig. 1 With $b=2, f_{2}=0.001$ and varing $f_{1}$, local bifurcation diagram are present: (a) $f_{1}=0.001$, (b) $f_{1}=0.002$, (c) $f_{1}=0.003$.

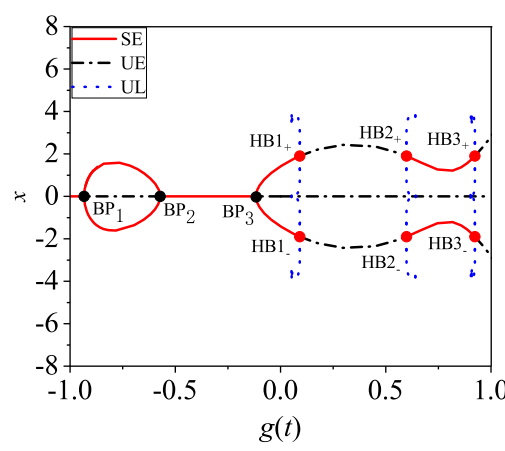

(a)

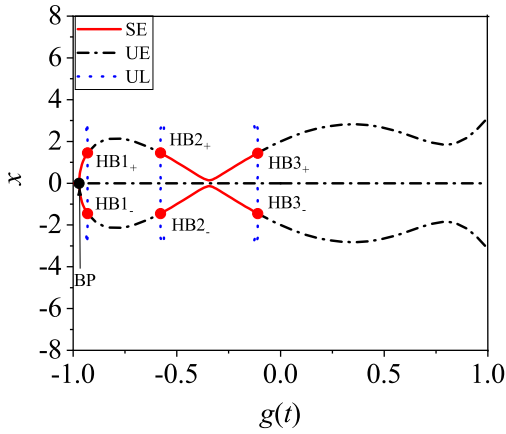

(b)

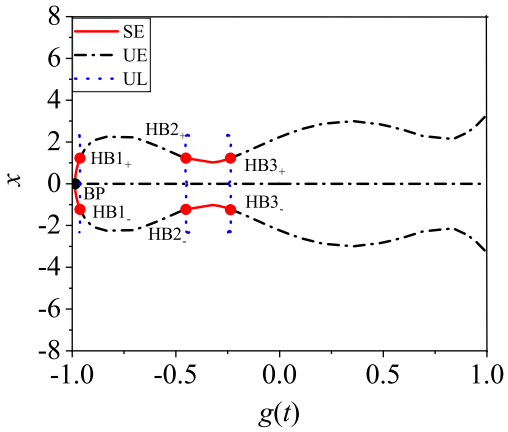

(c)

Fig. 2 With $f_{1}=0.002, f_{2}=0.01$ and varing $b$, local bifurcation diagram are present: (a) $b=2$, (b) $b=4$, (c) $b=5$.

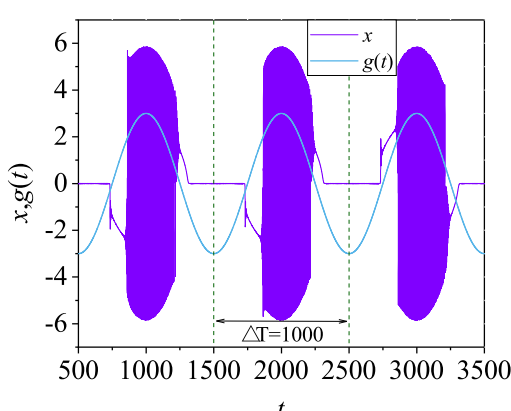

(a)

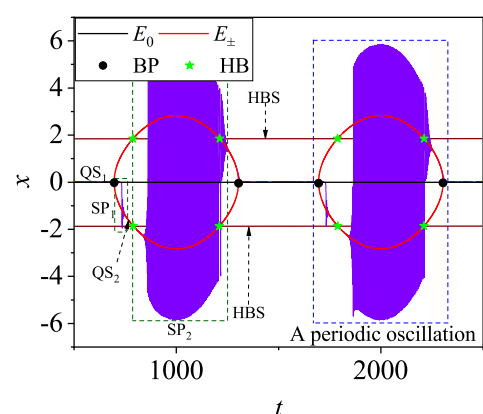

(b)

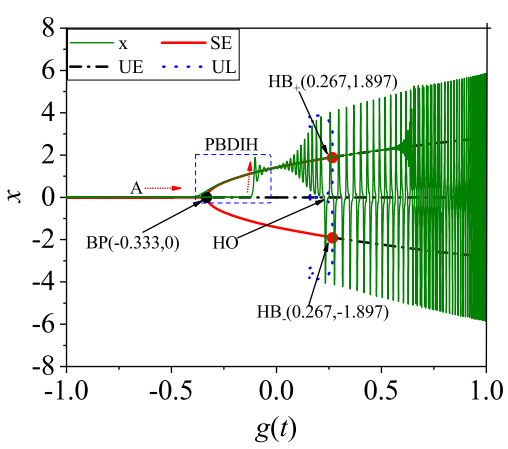

(c)

Fig. 3 Fast-slow dynamic for $f_{1}=f_{2}=0.001$ : (a) time series of $x$, (b) The superposition graph for the evolution of equilibrium point and time series of $x$ along with time $t$, (c) transformed phase diagram.

provide a variety of options for its application. Over the past few decades, a large number of BOs with different bifurcation mechanisms have been investigated. For instance, in some two-timescale system [13-16], fold/fold, fold/Hopf and symmetric Hopf/Hopf burster have been observed. Recently, more complex BOs are found on multi-timescale systems. Such as the turnover-of-pitchfork-hysteresis-induced and the compound pitchfork-hysteresis-induced bursting oscillations are demonstrated in [17]. Otherwise, some complex mixed-mode oscillations (MMOs) are revealed in [18, 19]. The discovery of this kind of BO provides a new di- rection for research. Although a substantial amount of research works has been carried out for multi-timescale systems, many complex BOs or MBCBOs still remain unexplored. This inspires our work on the analysis of dynamics of multi-timescale BOs.

Based on the above discussion, this work is motivated to investigate the MTDs of a novel parametrically and externally driven circuit system, which the original system belongs to the class of the Lorenz system family. It is noteworthy that the system exhibits complex MBCBOs. For instance, periodic, quasi-periodic and chaotic MBCBOs are 
uncovered, which are caused by the cascading of pitchfork bifurcation-delay-induced hysteresis (PBDIH) [20], homoclinic orbit (HO) and Hopf bifurcation (HB). Unfortunately, these complicated MBCBOs were never studied, and the mechanism of various modes of MBCBOs is not revealed in detail. Noticeably, the period of a single MBCBOs is determined by excitation at a small-frequency. Moreover, the effect of system parameters on dynamic is also thoroughly studied by adjusting the system parameters. Finally, an ana$\log$ circuit is designed to verify the above complex oscillation signal through circuit simulation and hardware experiments.

The rest of this paper is organized as follows. In Sec. 2, a non-autonomous system with parametric and external excitation is presented and the excitation transformation method is briefly introduced. In Sec. 3, stability and local bifurcation of the proposed 3D non-autonomous system are comprehensively investigated. In Sec. 4, the complex dynamic behaviors are studied by numerical simulation. Besides, circuit simulations and hardware experiments are performed to validate the numerical simulation in Sec. 5. Finally, conclusions are drawn in Sec. 6.

\section{Mathematical model and methodology}

\subsection{Mathematical model}

In 2007, Cai et al. obtained a new Lorenz-like chaotic system by modifying the third equation of Lorenz system [21], studied its basic dynamic characteristics in detail, and designed a single controller to control the system. In fact, this system inherits basic characteristics of Lorenz system, such as large Lyapunov exponent and butterfly chaotic attractor. Therefore, we choose the system as research object to study the multi-timescale dynamics of Lorenz system family, and its model is described by

$$
\left\{\begin{array}{l}
\dot{x}=a(y-x), \\
\dot{y}=x-x z+b y, \\
\dot{z}=x^{2}-c z,
\end{array}\right.
$$

where $x, y$, and $z$ represent three state variables, while $a, b$, and $c$ are positive constants. Based on system (1), a novel non-autonomous system with parametric and external excitation is proposed to model multi-timescale dynamics of Lorenz system family under periodic excitation. Different from the previous systems, in this system, parametric excitation and external excitation are introduced separately to two differential equations. Its mathematical model is depicted as,

$$
\left\{\begin{array}{l}
\dot{x}=a(y-x), \\
\dot{y}=w_{1} x-x z+b y, \\
\dot{z}=x^{2}-c z-w_{2},
\end{array}\right.
$$

where $w_{1}=\beta_{1} \cos \left(2 \pi f_{1} t\right)$ and $w_{2}=\beta_{2} \cos \left(2 \pi f_{2} t\right)$ are two excitation signals. If the variable of system (1) is regarded as a harmonic signal with continuous phase and random envelope change, then the MFOS defines the mean time derivative of the phase [22,23], as follows

$\bar{\omega}=\lim _{T \rightarrow \infty} \frac{2 \pi N(T)}{T}$,

where $N$ is the number of maxima or minima in the time $T$. When the system (1) parameters are set as $a=10, b=2$ and $c=1$, the MFOS $\bar{\omega}_{0}$ can be calculated as 16.71, which means that $\bar{f}_{0}=\bar{\omega}_{0} /(2 \pi) \approx 2.659$. As a result, when the frequency of the two excitation in the system (2) meets $0<$ $f_{1,2} \ll 1$ (that is, far less than $\bar{f}_{0}$ ), the order gap is formed in the time scale of the improved non-autonomous system, which inevitably lead the system to generate complicated dynamics.

\subsection{Methodology}

For a fast - slow dynamical system with two slowly varying excitation, its dynamic behavior can be described by

$\dot{x}(t)=f\left(x(t), w_{1}(t), w_{2}(t)\right)$,

where vector $x(t) \in R^{n}$ stands for fast subsystem. Usually, $w_{1}(t)=\beta_{1} \cos \left(2 \pi f_{1} t\right)$ and $w_{2}(t)=\beta_{2} \cos \left(2 \pi f_{2} t\right)$ is regarded as a slow subsystem. It is considered that the classical fastslow analysis method [24] cannot be used to analyze the dynamics of the fast subsystem when there are multiple variables in the slow subsystem. Therefore, to address this problem, it is necessary to introduce a generalized slow variable $g(t)$ by the excitation transformation method [18], so that $w_{1}(t), w_{2}(t)$ and $g(t)$ exist uniformly in the system in the form of independent variables and dependent variables of the function.

Next, brief introduction of the excitation transformation method is given as below. Assuming that two slow variables are $w_{1}=\cos (a t)$ and $w_{2}=\cos (b t)$, and $n=a / b$, where $n$ is integer, rational or irrational number. Let's take $n$ as an integer to illustrate the excitation transformation in detail. For the other two cases, $[18,25]$ give the detailed explanations. Firstly, according to Moivre's formula [26], the following relationships can be obtained,

$(\cos (x)+i \sin (x))^{n}=\cos (n x)+i \sin (n x)$.

Then, by expanding the left side of polynomial (5), we can get

$$
\begin{aligned}
(\cos (x)+i \sin (x))^{n}= & C_{n}^{0} \cos ^{n}(x)+C_{n}^{1} \cos ^{n-1}(x)(i \sin (x)) \\
& +\ldots+C_{n}^{n-1} \cos (x)(i \sin (x))^{n-1} \\
& +C_{n}^{n}(i \sin (x))^{n}
\end{aligned}
$$




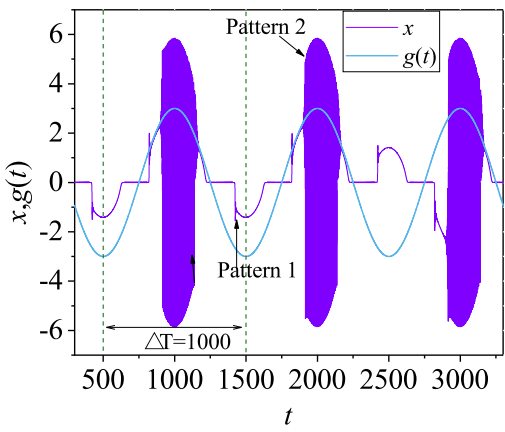

(a)

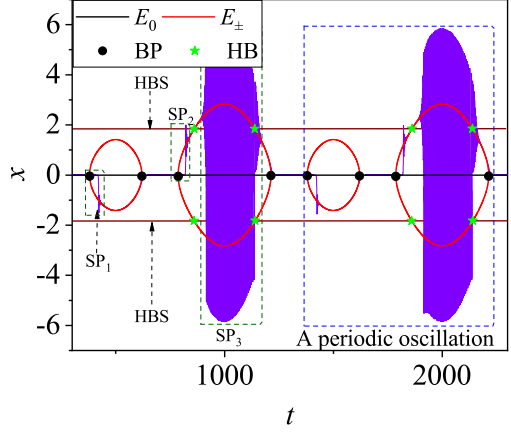

(b)

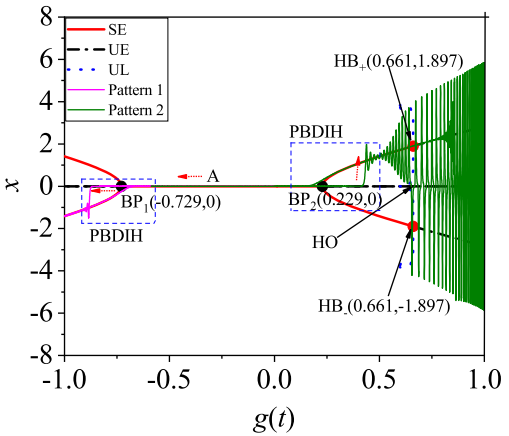

(c)

Fig. 4 Fast-slow dynamic for $f_{1}=0.002, f_{2}=0.001$ : (a) time series of $x$, (b) The superposition graph for the evolution of equilibrium point and time series of $x$ along with time $t$, (c) transformed phase diagram.

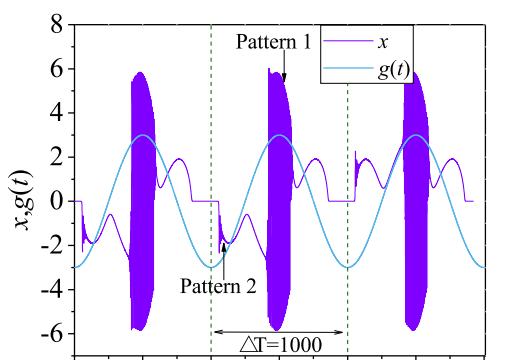

1500200025003000350040004500

(a)

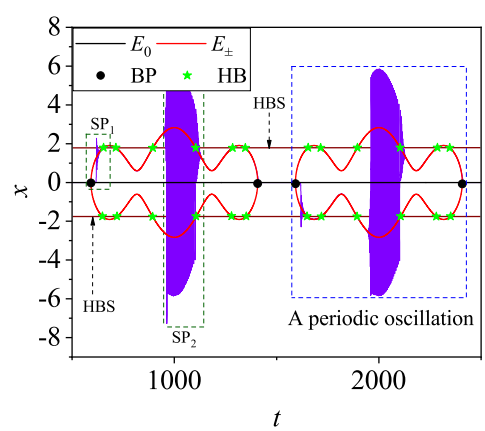

(b)

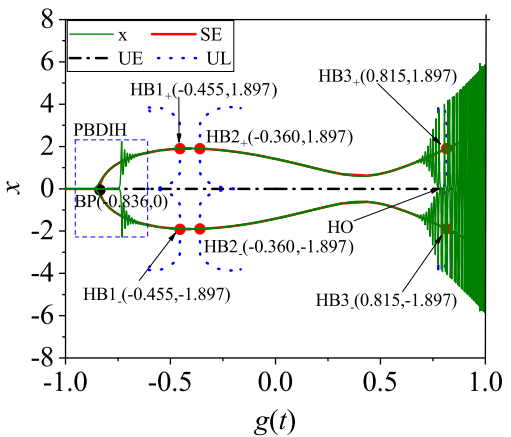

(c)

Fig. 5 Fast-slow dynamic for $f_{1}=0.003, f_{2}=0.001$ : (a) time series of $x$, (b) The superposition graph for the evolution of equilibrium point and time series of $x$ along with time $t$, (c) transformed phase diagram.

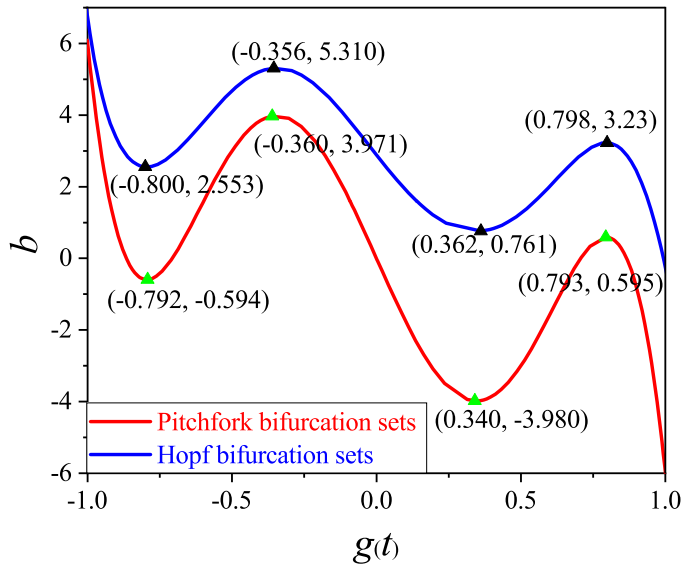

Fig. 6 The two-parameter bifurcation sets of $b$ and $g(t)$ for $a=10$, $c=1, f_{1}=0.002$ and $f_{2}=0.01$.

Comparing the right side of (5) and (6), we have

$$
\begin{aligned}
\cos (n x)= & C_{n}^{0} \cos ^{n}(x)-C_{n}^{2} \cos ^{n-2}(x) \sin ^{2}(x) \\
& +C_{n}^{4} \cos ^{n-4}(x) \sin ^{4}(x) \\
& -\ldots+C_{n}^{m} \cos ^{n-m}(x)(i \sin (x))^{m},
\end{aligned}
$$

Using trigonometric equations, (7) can be rewritten as,

$$
\begin{aligned}
\cos (n x)= & C_{n}^{0} \cos ^{n}(x)-C_{n}^{2} \cos ^{n-2}(x)\left(1-\cos ^{2}(x)\right) \\
& +C_{n}^{4} \cos ^{n-4}(x)\left(1-\cos ^{2}(x)\right)^{2}-\ldots \\
& +C_{n}^{m} \cos ^{n-m}(x)\left(1-\cos ^{2}(x)\right)^{m / 2}=g(\cos (x)) .
\end{aligned}
$$

where $m \leq n$ and $m$ is even.

If there exists an integer $k$, such that $a=p k$ and $b=q k$ are true. Depending on (8), we can get

$$
\begin{aligned}
w_{1}= & C_{p}^{0} \cos ^{p}(k t)-C_{p}^{2} \cos ^{p-2}(k t)\left(1-\cos ^{2}(k t)\right) \\
& +C_{p}^{4} \cos ^{p-4}(k t)\left(1-\cos ^{2}(k t)\right)^{2} \\
& -\ldots+C_{p}^{m} \cos ^{n-m}(k t)\left(1-\cos ^{2}(k t)\right)^{m / 2}=\cos (a t) \\
w_{2}= & C_{q}^{0} \cos ^{q}(k t)-C_{q}^{2} \cos ^{q-2}(k t)\left(1-\cos ^{2}(k t)\right) \\
& +C_{q}^{4} \cos ^{q-4}(k t)\left(1-\cos ^{2}(k t)\right)^{2} \\
& -\ldots+C_{q}^{m} \cos ^{q-m}(k t)\left(1-\cos ^{2}(k t)\right)^{m / 2}=\cos (b t)
\end{aligned}
$$

It follows that the periodic excitation $w_{1}$ and $w_{2}$ in the system can be replaced by a polynomial function of $\cos (k t)$. 


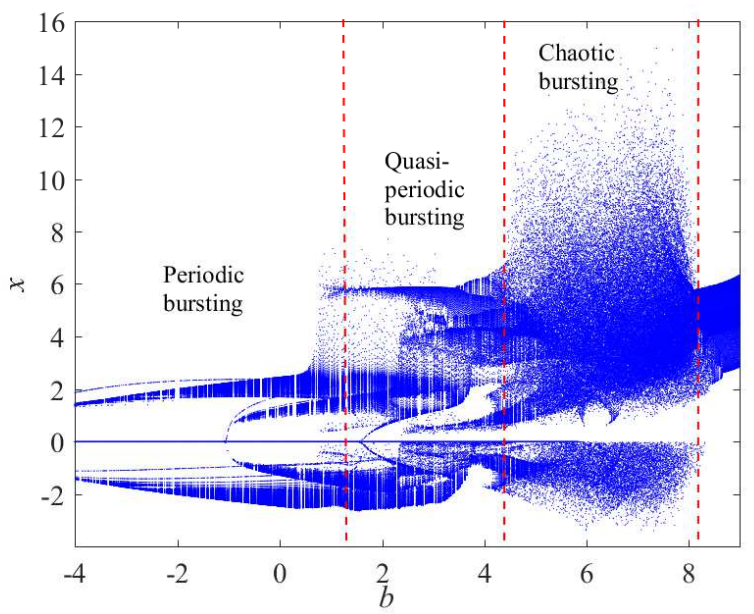

(a)

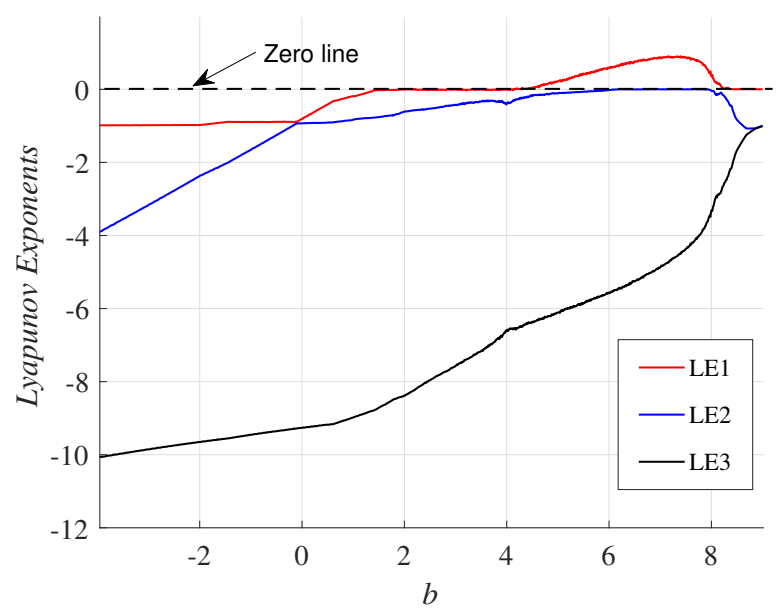

(b)

Fig. 7 With $f_{1}=0.002$ and $f_{2}=0.01$, the dynamics of system (1) with $b$ varying: (a) bifurcation diagram, (b) Lyapunov exponents.

It turns out that there is only a uniformly generalized slow variable in the system, $\cos (k t)$, denoted by $g(t)$.

Secondly, according to different excitation frequencies, the system can be divided into slow subsystem $g(t)$, and generalized fast subsystem in the followling three cases:

i) When $f_{1}>f_{2}$, set $g(t)=w_{2}(t), f(g(t))=w_{1}(t)$, then fast subsystem can be rewritten as

$$
\left\{\begin{array}{l}
\dot{x}=a(y-x), \\
\dot{y}=f(g(t)) x-x z+b y, \\
\dot{z}=x^{2}-c z-g(t),
\end{array}\right.
$$

ii) When $f_{1}<f_{2}$, set $g(t)=w_{1}(t), w_{2}(t)=f(g(t))$, then fast subsystem can be rewritten as

$$
\left\{\begin{array}{l}
\dot{x}=a(y-x), \\
\dot{y}=g(t) x-x z+b y, \\
\dot{z}=x^{2}-c z-f(g(t)),
\end{array}\right.
$$

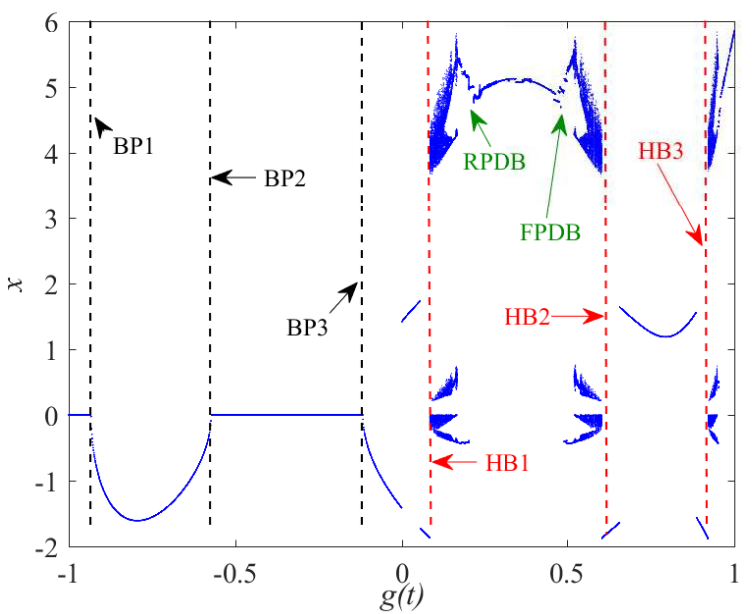

(a)

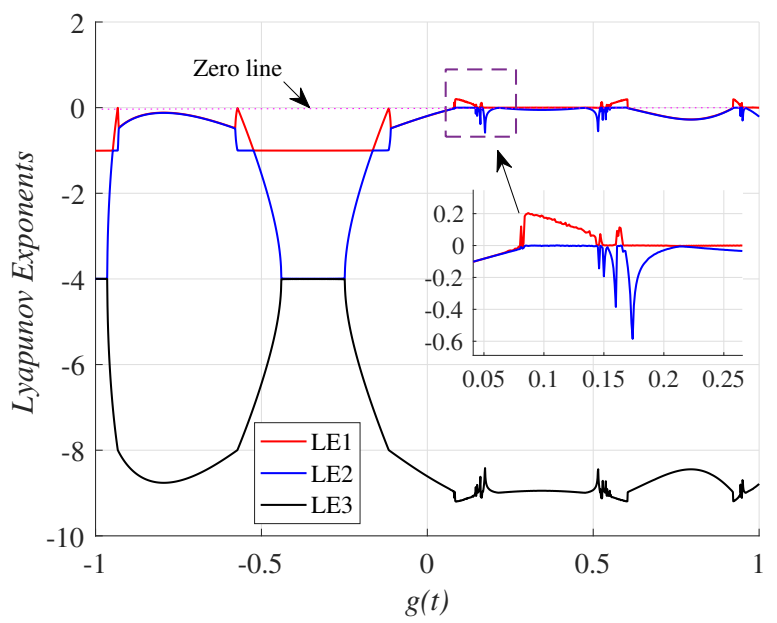

(b)

Fig. 8 With $b=2, f_{1}=0.002$ and $f_{2}=0.01$, the dynamics of system (1) with $g(t)$ varying: (a) bifurcation diagram, (b) Lyapunov exponents.

iii) When $f_{1}=f_{2}$, set $g(t)=w_{1}(t)=w_{2}(t)$, then fast subsystem can be rewritten as

$$
\left\{\begin{array}{l}
\dot{x}=a(y-x) \\
\dot{y}=g(t) x-x z+b y \\
\dot{z}=x^{2}-c z-g(t)
\end{array}\right.
$$

It is concluded that slow subsystem can be replaced by $g(t)$ with only one slow-varying excitation. In addition, the excitation amplitude $\beta_{1}$ and $\beta_{2}$ are usually taken as a fixed parameters when performing fast-slow analysis. Note that here the excitation amplitude is generally 1 , and typical examples are given in Table 1 and 2. Consequently, it can be seen from (9) that the high-frequency excitation can be replaced by the polynomial of low-frequency excitation. Next, these complicated dynamics are analyzed theoretically and numerically. 


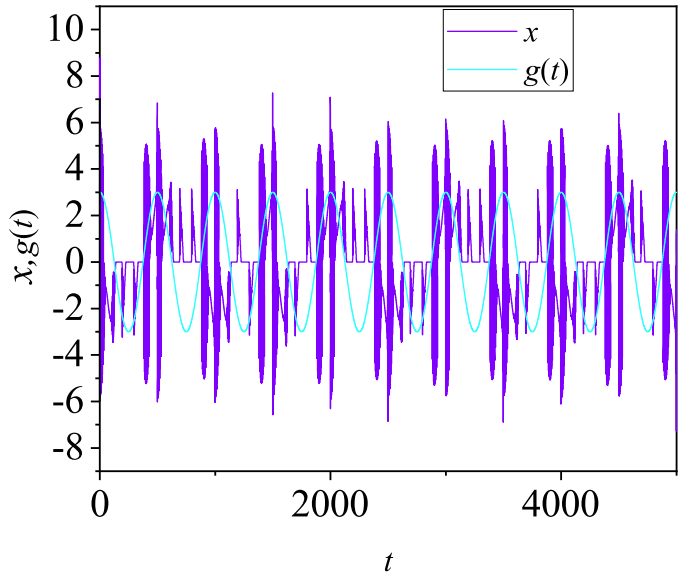

(a)

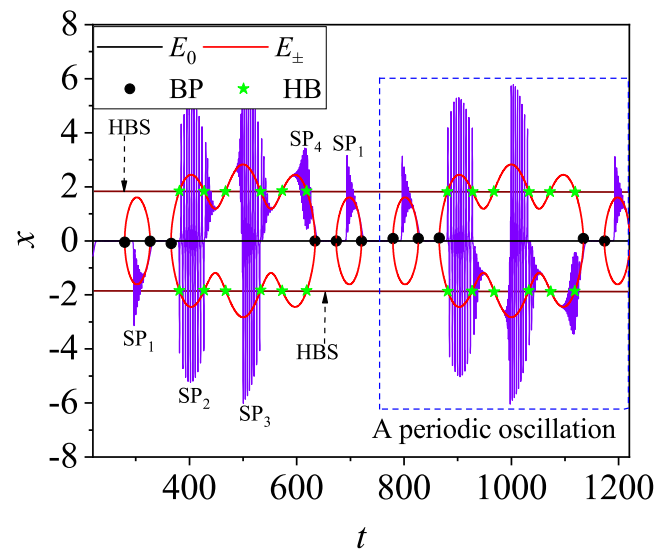

(c)

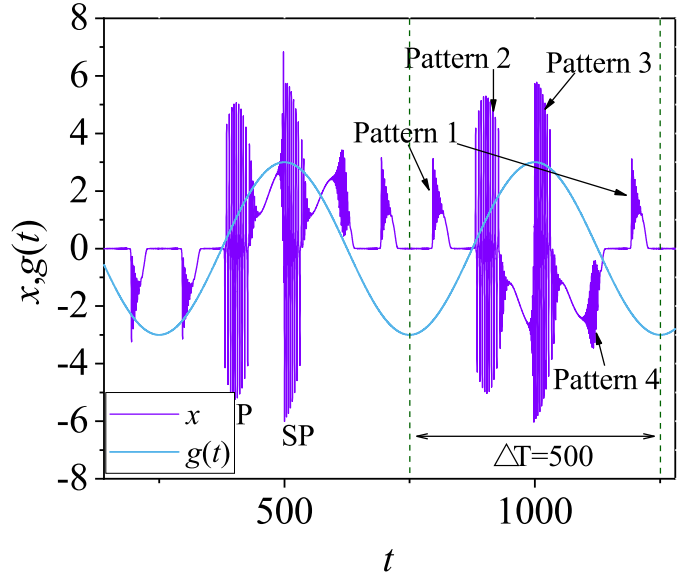

(b)

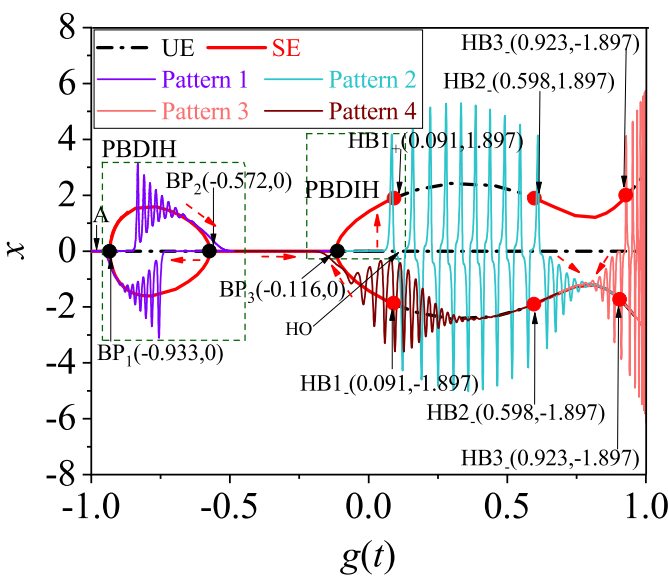

(d)

Fig. 9 Fast-slow dynamic for $b=2$ : (a) whole time series of $x$ and $g(t)$, (b) Local enlarged time series of $x$ and $g(t)$, (c) The superposition graph for the evolution of equilibrium point and time series of $x$ along with time $t$, (d) transformed phase diagram.

Table 1 If $f_{1}<f_{2}$, the relation between $g(t)$ and $f(g(t))$

\begin{tabular}{ccc}
\hline \hline$f_{1} / f_{2}$ & $g(t)$ & $f(g(t))$ \\
\hline 1 & $w_{1}$ or $w_{2}$ & $g(t)$ \\
$1 / 2$ & $w_{1}$ & $2 g(t)^{2}-1$ \\
$1 / 3$ & $w_{1}$ & $4 g(t)^{3}-3 g(t)$ \\
$1 / 4$ & $w_{1}$ & $8 g(t)^{4}-8 g(t)^{2}+1$ \\
$1 / 5$ & $w_{1}$ & $16 g(t)^{5}-20 g(t)^{3}+5 g(t)$ \\
$\vdots$ & $\vdots$ & $\vdots$ \\
\hline \hline
\end{tabular}

\section{Local bifurcation and stability analysis of equilibrium point}

For a dynamic system, the characteristics of the equilibrium point affect the complex dynamics to a large extent. For example, unstable saddle-focus may lead to the formation of chaotic attractors [27], the changes of equilibrium branch structure and properties lead to the emergence of all kinds
Table 2 If $f_{1}>f_{2}$, the relation between $g(t)$ and $f(g(t))$

\begin{tabular}{ccc}
\hline \hline$f_{1} / f_{2}$ & $g(t)$ & $f(g(t))$ \\
\hline 1 & $w_{1}$ or $w_{2}$ & $g(t)$ \\
$2 / 1$ & $w_{2}$ & $2 g(t)^{2}-1$ \\
$3 / 1$ & $w_{2}$ & $4 g(t)^{3}-3 g(t)$ \\
$4 / 1$ & $w_{2}$ & $8 g(t)^{4}-8 g(t)^{2}+1$ \\
$5 / 1$ & $w_{2}$ & $16 g(t)^{5}-20 g(t)^{3}+5 g(t)$ \\
$\vdots$ & $\vdots$ & $\vdots$ \\
\hline
\end{tabular}

of bifurcations [28]. Therefore, this section will focus on the characteristics of the equilibrium point and the local bifurcation. First of all, suppose that

$\dot{x}=\dot{y}=\dot{z}=0$,

it is obvious that the equilibrium points of system (2) are determined by the following formula

$-x^{3}+q x=0$, 


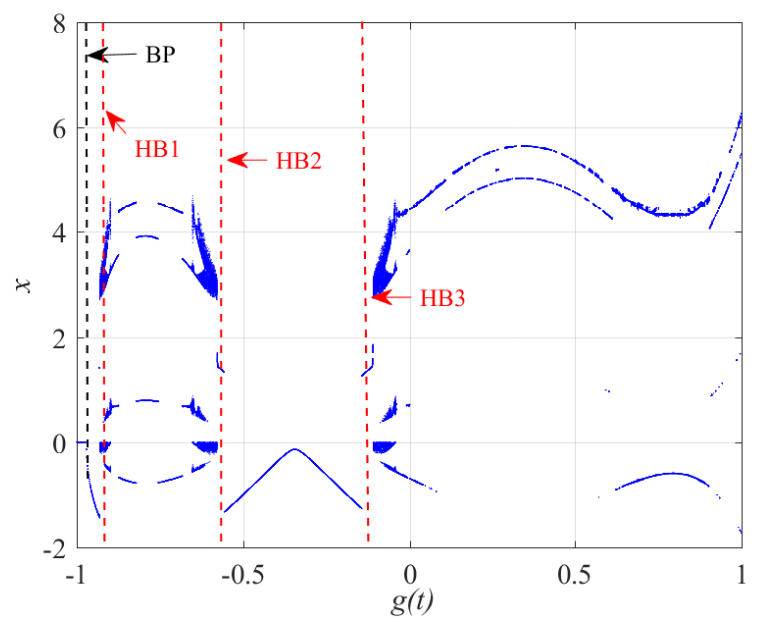

(a)

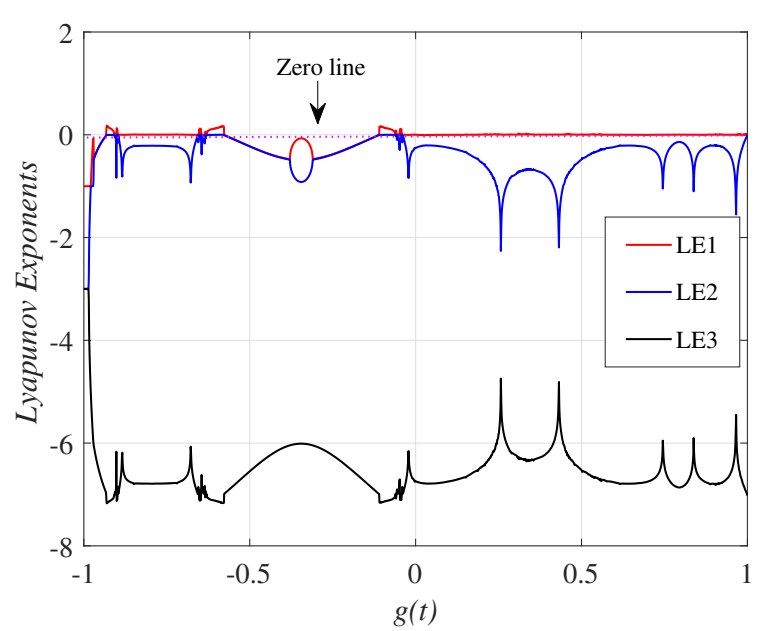

(b)

Fig. 10 With $b=4, f_{1}=0.002$ and $f_{2}=0.01$, the dynamics of system (1) with $g(t)$ varying: (a) bifurcation diagram, (b) Lyapunov exponents.

where $q=c w_{1}+w_{2}+b c$. Therefore, it is necessary to analyze the existence of equilibrium points in two cases:

i) If $q \leq 0$, then system (1) has only $E_{0}=\left(0,0,-w_{2} / c\right)$;

ii) If $q>0$, then system (1) has $E_{0}$ and $E_{ \pm}=( \pm \sqrt{q}, \pm \sqrt{q}$, $\left.w_{1}+b\right)$.

It can be found that the existence of the equilibrium point is closely related to $w_{1}$ and $w_{2}$, which means that the trajectory of equilibrium point (TEP) will undergo a complicated change when the two slowly varying excitations change at the same time.

The local stability of the equilibrium point can be determined by eigenvalues of following Jacobian matrix

$J=\left(\begin{array}{lll}\partial \dot{x} / \partial x & \partial \dot{x} / \partial y & \partial \dot{x} / \partial z \\ \partial \dot{y} / \partial x & \partial \dot{y} / \partial y & \partial \dot{y} / \partial z \\ \partial \dot{z} / \partial x & \partial \dot{z} / \partial y & \partial \dot{z} / \partial z\end{array}\right)=\left(\begin{array}{ccc}-a & a & 0 \\ w_{1}-z & b & -x \\ 2 x & 0 & -c\end{array}\right)$
The characteristic equation of (10) can be found as

$f(\lambda)=(\lambda-J)=\lambda^{3}+m_{1} \lambda^{2}+m_{2} \lambda+m_{3}=0$,

in which

$\left\{\begin{array}{l}m_{1}=a+c-b, \\ m_{2}=a z+a c-a w_{1}-a b-b c, \\ m_{3}=a c z-a c w_{1}-a b c+2 a x^{2} .\end{array}\right.$

According to the Routh-Hurwitz criterion [29], if the following conditions are met

$\left\{\begin{array}{l}m_{1}>0 \\ m_{1} m_{2}-m_{3}>0 \\ m_{3}>0\end{array}\right.$

the equilibrium point is locally asymptotically stable. Otherwise, it is unstable. Now, a specific example is given to illustrate the characteristics of equilibrium change. Letting $a=10, b=2, c=1, \beta_{1}=\beta_{2}=3, f_{1}=0.003$ and $f_{2}=0.001$, then $w_{2}=g(t), w_{1}$ can be replaced by $4 g(t)^{3}-3 g(t)$ based on Table 2. Next, the corresponding equilibrium points $E_{0}$ and $E_{ \pm}$are substituted into (11), and the following characteristic equation can be obtained as,

$$
\begin{aligned}
f_{0}(\lambda)= & \lambda^{3}+9 \lambda^{2}+\left(60 g(t)-120 g(t)^{3}-12\right) \lambda \\
& 60 g(t)-120 g(t)^{3}-20=0,
\end{aligned}
$$

and

$$
\begin{aligned}
f_{ \pm}(\lambda)= & \lambda^{3}+9 \lambda^{2}+8 \lambda \\
& 240 g(t)^{3}-120 g(t)+40=0 .
\end{aligned}
$$

According to the characteristic equations of (16) and (17) with higher degree polynomials, it can be known that there are many local bifurcations under the control of the slowly varying excitation $w_{1}$ and $w_{2}$. And there are mainly two kinds of bifurcations:

i) Pitchfork bifurcation[30].

ii) Hopf bifurcation [31].

On the one hand, if the following condition is true,

$60 g(t)-120 g(t)^{3}-20=0$,

i.e. $g(t)=-0.836$, the characteristic equation has a zero eigenvalue, which proves that this equilibrium point is a nonhyperbolic equilibrium point. When $g(t)$ passes through 0.8362 slowly, the stable equilibrium (SE) point is split into two SE points and one unstable equilibrium (UE) point, leading to the emergence of pitchfork bifurcation (BP). The corresponding TEP is plotted in Fig. 1(c), it also can be seen that the bifurcation point is supercritical.

On the other hand, if the following condition is satisfied,

$-240 g(t)^{3}+120 g(t)+32=0$, 


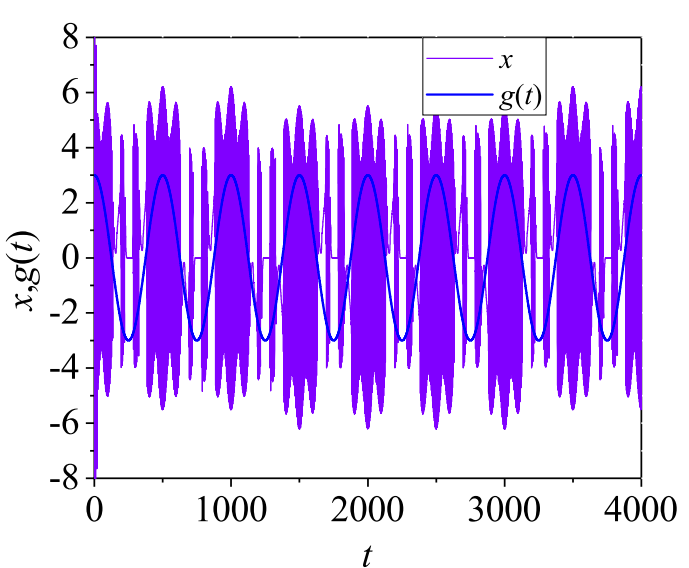

(a)

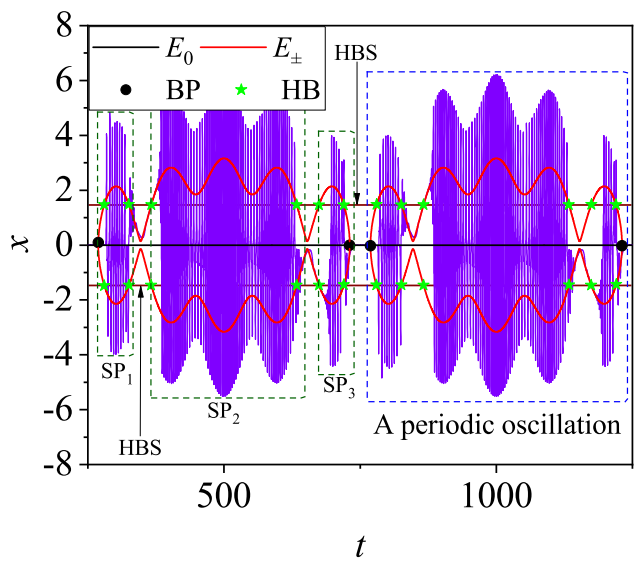

(c)

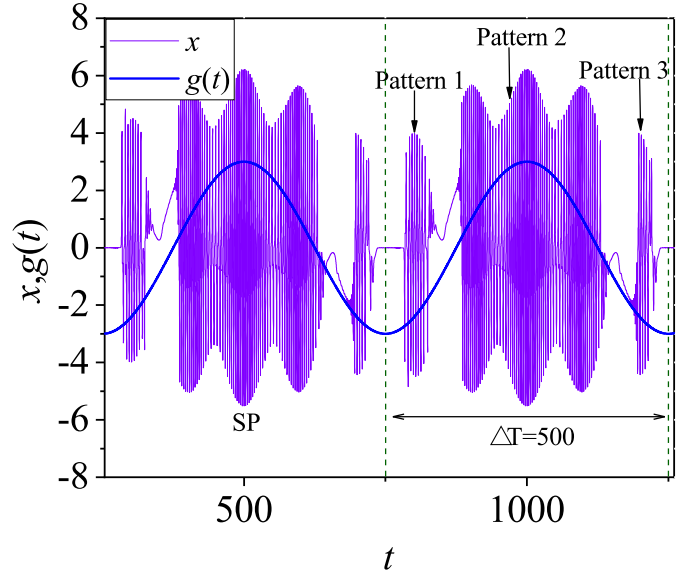

(b)

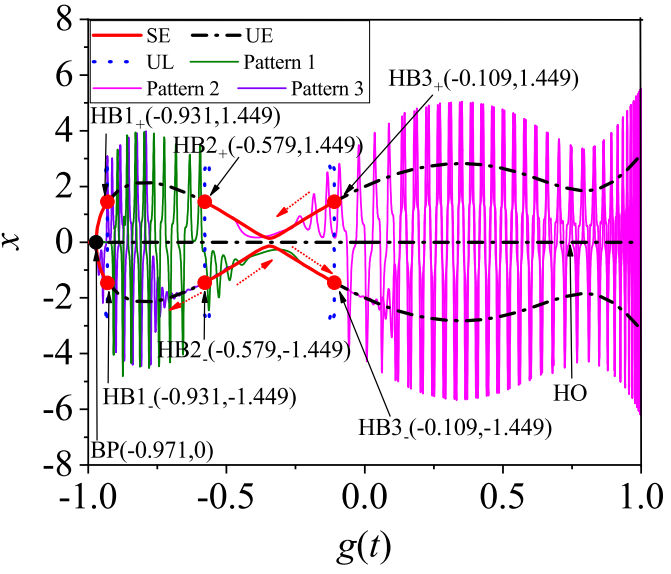

(d)

Fig. 11 Fast-slow dynamic for $b=4$ : (a) whole time series of $x$ and $g(t)$, (b) Local enlarged time series of $x$ and $g(t)$, (c) The superposition graph for the evolution of equilibrium point and time series of $x$ along with time $t$, (d) transformed phase diagram.

i.e. $g(t)=0.815,-0.455$ and -0.360 , the characteristic equation has a pair of purely imaginary eigenvalues, which implies that the TEPs and periodic solutions meet at a bifurcation point. Thus, HB may occur, which leads to periodic oscillation and unstable limitcycle (UL). Besides, its period is determined by the imaginary part of the eigenvalue.

Following the above analysis, the TEPs under the condition of other parameters are also plotted in Fig. 1 and Fig. 2 by utilizing XPPAUT. It is verified that the system has a variety of TEPs for different system parameters and the excitation frequency. Besides, the local bifurcation points have an increasing trend with the increase of frequency ratio. This behavior is likely to lead to complex dynamical behavior.

\section{Numerical results of dynamics and fast-slow analysis}

In this section, a variety of complex oscillatory behaviors of system (2) will be simulated by using MATLAB. Besides, the ODE45 algorithm is used to solve differential equations, in which the time step is set as 0.01 . Furthermore, various MBCBOs of system (2) under the different frequency ratio are studied separately. And the effect of system parameters on dynamical behaviors is also studied.

4.1 The delayed-pitchfork/Hopf/homoclinic induced periodic bursting

Setting $f_{1}=f_{2}=0.001, \beta_{1}=\beta_{2}=3, a=10, b=2, c=1$, and the initial condition is $(0.1,0,0)$. The simulation time is set as 5000. Dynamic and fast-slow analyses are presented in Fig. 3. It can be seen from Fig. 3(b) that the evolution of $\mathrm{BO}$ and equilibrium point is basically consistent, and the intersection of Hopf bifurcation set (HBS) and $E_{ \pm}$represents HB point.

The formation mechanism of BO is further analyzed by combining Fig. 3(b) and 3(c). Suppose the trajectory starts at $\mathrm{QS}_{1}$ (i.e. the point $\mathrm{A}$ in Fig. 3(c)) under the control of generalized slow variable $g(t)$. When the trajectory passes 


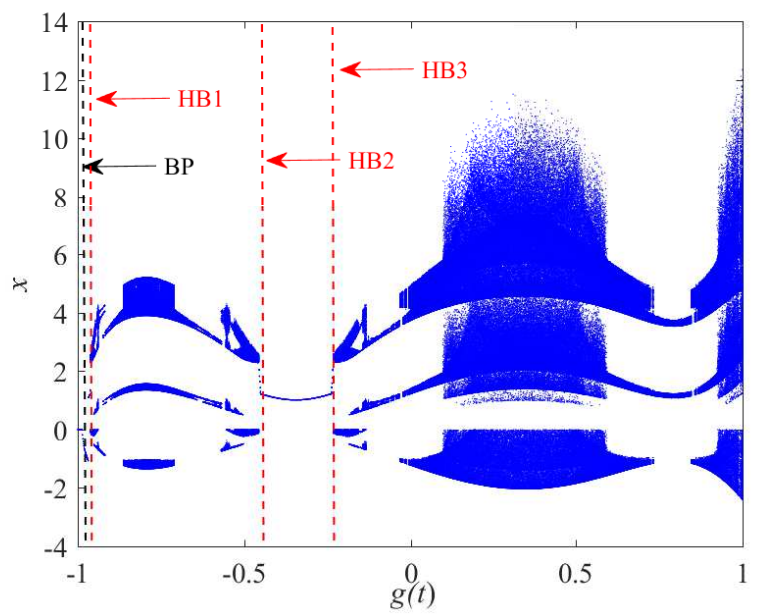

(a)

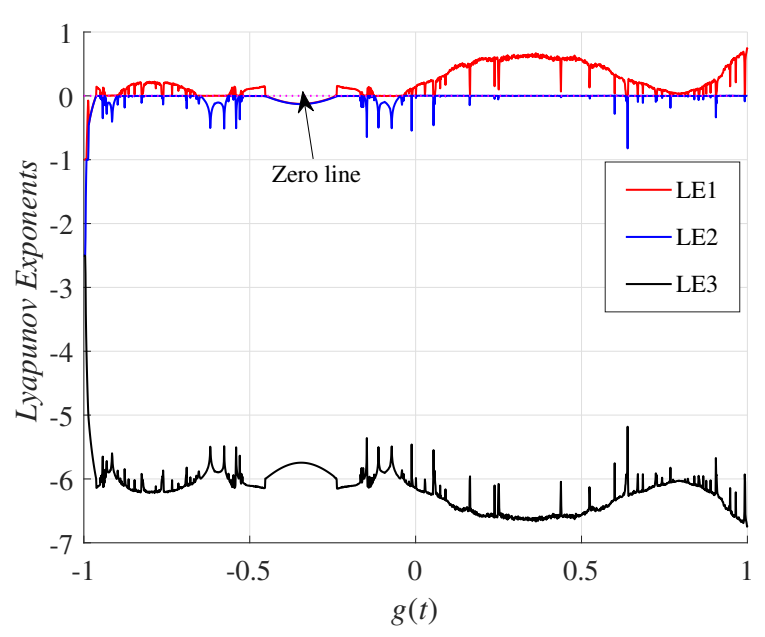

(b)

Fig. 12 With $b=5, f_{1}=0.002$ and $f_{2}=0.01$, the dynamics of system (2) with $g(t)$ varying: (a) bifurcation diagram, (b) Lyapunov exponents.

through BP slowly, the PBDIH is formed, which results in the trajectory going through a period delay before entering $\mathrm{SP}_{1}$. Then, because the equilibrium point is always stable, the trajectory gradually converges to $\mathrm{QS}_{2}$. Following the trajectory passes through the $\mathrm{HB}_{+}$point slowly, which leads to $\mathrm{SP}_{2}$ and the amplitude increases gradually. At the same time, two symmetrical saddle-focus appear in the system. Therefore, HO is formed, and the trajectory begins to oscillate back and forth between the two saddle-focus. Finally, the trajectory returns to $\mathrm{HB}_{+}$again, leading the trajectory returns to $\mathrm{QS}_{1}$ until $\mathrm{BP}$. Consequently, the oscillation behavior is named as the delayed-pitchfork/Hopf/homoclinic induced periodic bursting.
4.2 The first cascading periodic bursting induced by delayed-pitchfork/Hopf/homoclinic

Leave the other parameters unchanged and increase $f_{1}$ to 0.002. Then dynamic and fast-slow analysis is plotted in Fig. 4. It is seen that the period of a single cascaded bursting oscillation is determined by excitation at a small-frequency and is equal to $\triangle T=1 / f_{1,2}$.

Fig. 4 shows that there is a cascade of two patterns within a single bursting oscillation period. In fact, there is one more PBDIH induced bursting [17] comparing with the delayedpitchfork/Hopf/homoclinic induced periodic bursting. Therefore, the mechanism is similar to the former, refer to the previous subsection.

4.3 The second cascading periodic bursting induced by delayed-pitchfork/Hopf/homoclinic

Increase continuously $f_{1}$ to 0.003 . Dynamic and fast-slow analysis is shown in Fig. 5. Besides, Fig. 1(c) shown that the TEP has changed tremendously comparing with the previous two patterns. The most prominent feature is the bending of the TEP, and there are three pairs of HB.

Actually, the mechanism of bifurcation is the same as The first cascading periodic bursting induced by delayedpitchfork/Hopf/homoclinic. From Fig. 5(b) and 5(c) we can also know that when the trajectory gets to the BP point, it experiences a PBDIH before entering SP. The main difference is that there are continuous twists and turns, and the state of system (2) does not change at all when the trajectory passes through the $\mathrm{HB} 1_{+}$and $\mathrm{HB} 2+$ points, and stay in QS. Subsequently, system (2) enters the periodic SP because the equilibrium is unstable. Finally, trajectory passes through the $\mathrm{HB} 3_{+}$, the trajectory returns to QS. Therefore, the oscillation is also considered as the second cascading periodic bursting induced by delayed-pitchfork/Hopf/homoclinic.

4.4 The first delayed-pitchfork/Hopf/homoclinic cascade induced quasi-periodic bursting

A large number of numerical results show that the effects of system parameters on the system dynamics are very similar under different frequency ratios. In this subsection, two determined frequencies are selected to study the system dynamics. Now setting $a=10, c=1, \beta_{1}=\beta_{2}=3, f_{1}=0.002$, $f_{2}=0.01$, and the initial condition is $(0.1,0,0)$. The simulation time is set as 5000. Besides, $w_{1}=g(t), w_{2}=f(g(t))=$ $16 g(t)^{5}-20 g(t)^{3}+5 g(t)$. Then the two-parameter local bifurcation sets for $g(t)$ and $b$ can be obtained, and shown in Fig. 6. It is seen that the number and location of BP and HB will change with $b$ and $g(t)$. 


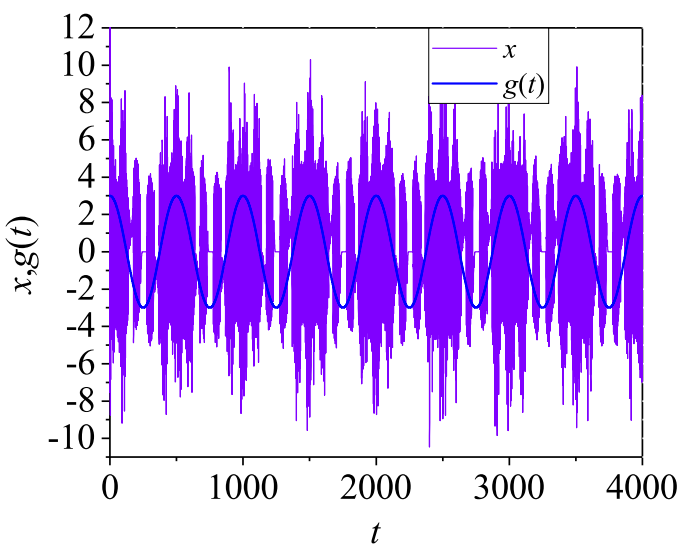

(a)

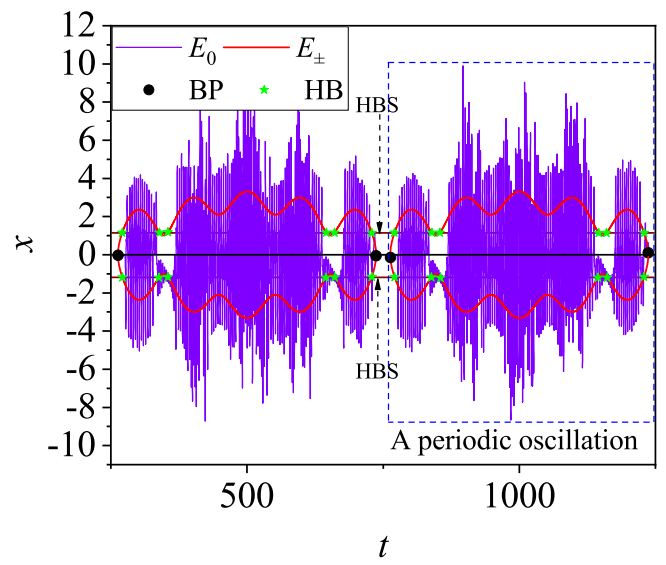

(c)

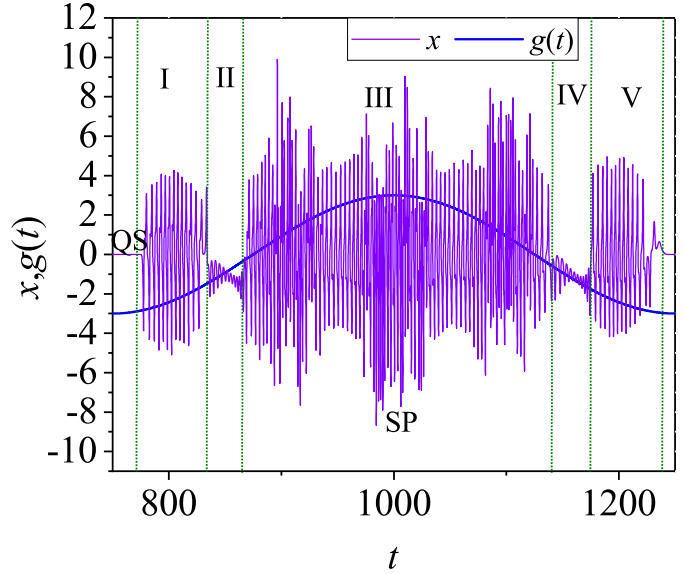

(b)

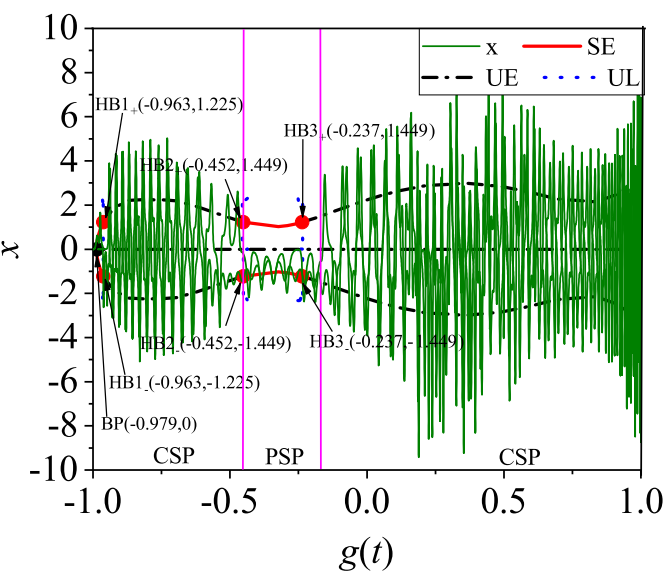

(d)

Fig. 13 Fast-slow dynamic for $b=5$ : (a) whole time series of $x$ and $g(t)$, (b) Local enlarged time series of $x$ and $g(t)$, (c) The superposition graph for the evolution of equilibrium point and time series of $x$ along with time $t$, (d) transformed phase diagram.

\subsubsection{Bifurcation diagram and Lyapunov exponents analysis}

To further study the effect of parameter $b$, other parameters are fixed and $b$ is used as a variable parameter to draw the bifurcation diagram and Lyapunov exponents of single parameter, results are shown in Fig. 7. It can be seen that the dynamical behaviors reflected by the trajectories in Fig. 7(a) and 7(b) agree with each other, and system (2) exhibits complex oscillatory behaviors. Firstly, the system oscillates periodically when parameter $b$ varies in the range of $(-4,3)$. Subsequently, in the range of $(3,4.4),(8.3,9)$ and $(4.4,8.3)$, the system exhibits quasi-periodic and chaotic oscillation respectively, which means that the system leads to chaos through the quasi-periodic route. Finally, the system exits chaos by inverse quasi-periodic. Furthermore, it is obvious that the trajectory jumps around the zero-point within the region $(-4,8)$, which means that complex bursting oscillations may occur.
Setting $b=2$ and using $g(t)$ as a variable parameter, the bifurcation diagram and Lyapunov exponents of single parameter are shown in Fig. 8. The results show that under the control of the slowly varying parameter $g(t)$, the system experiences all kinds of attractors, including periodic, quasi-periodic and chaotic attractors. Besides, some interesting bifurcations have also been observed, such as BP, $\mathrm{HB}$, forward period-doubling bifurcation (FPDB) and reverse period-doubling bifurcation (RPDB). Therefore, it is also confirmed that the bursting oscillation is caused by the switching between different attractors. Also, it is interesting to observe from LEs in Fig. 7(b) that the overall behavior of the system presents a quasi-periodic state. However, from the time series in Fig. 9(a), it seems to be in chaos.

\subsubsection{Mechanism analysis}

For a better understanding of the bursting process, Dynamic and fast-slow analysis are presented in Fig. 9. It is shown from Fig. 9(b) and Fig. 9(c) that complex phenomenon of 


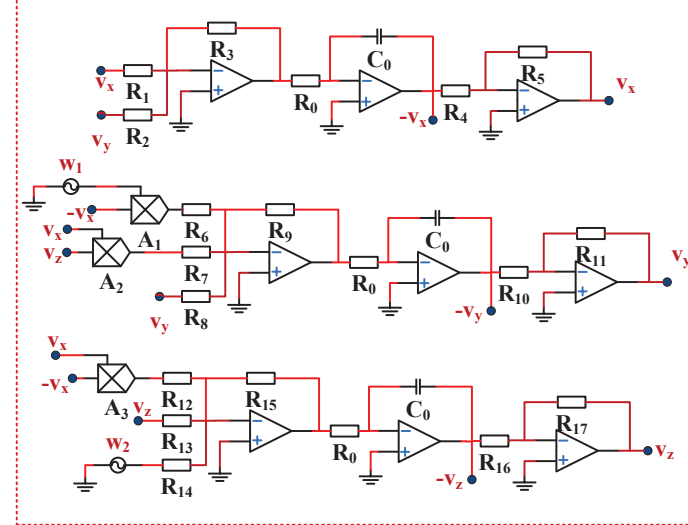

(a)

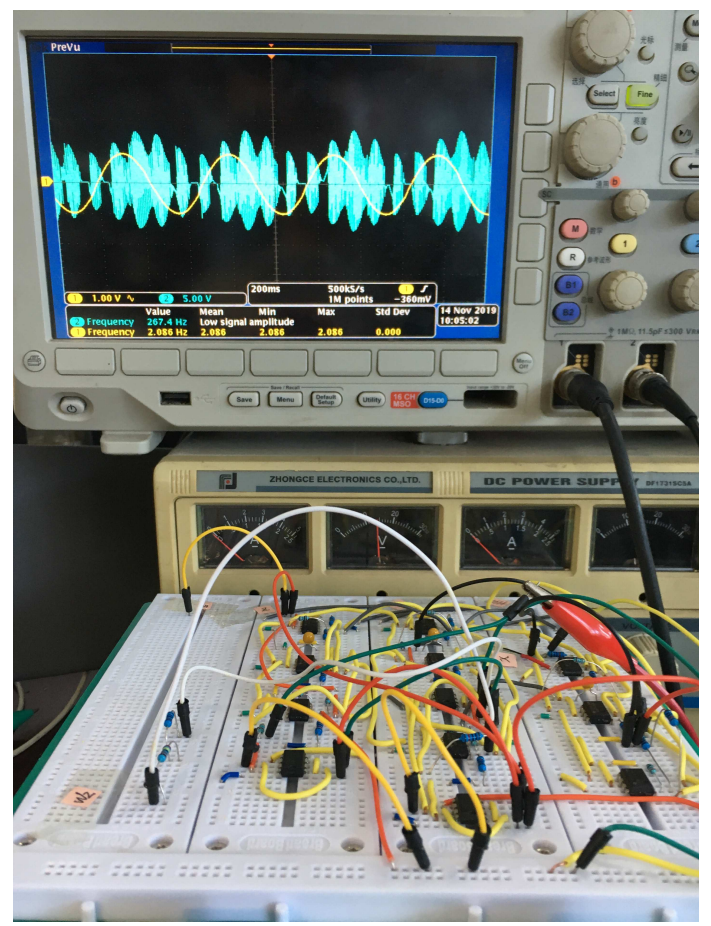

(b)

Fig. 14 Circuit experimental verification: (a) the circuit schematic of system (1), (b) experimental setup: breadboard, power supply and oscilloscope.

spiking transfer under the control of HB. Moreover, the trajectory of purple, light blue, red and brown represents four different patterns of spiking in Fig. 9(d).

Assuming that the trajectory starts to oscillate at point A. Firstly, the trajectory experiences the first pattern of $\mathrm{BO}$ caused by $\mathrm{PBDIH}$ when it passes through $\mathrm{BP}_{1}$ and the oscillation trajectory is represented by the purple. However, the second pattern of BO is more complicated comparing with the former. When $g(t)$ moves to $\mathrm{BP}_{3}$, the trajectory enters the second pattern of $\mathrm{BO}$, at this time, the $\mathrm{SP}_{2}$ is formed. The existence of $\mathrm{HB}_{1}$ causes the saddle-foci equilibrium point to appear, and then the trajectory oscillates between two saddle-foci and is connected by HO. Finally, the system returns to QS through $\mathrm{HB}_{2}$. Next, the trajectory follows the SE passes through $\mathrm{HB}_{3}$, and the trajectory enters into $\mathrm{SP}_{3}$. Due to the existence of $\mathrm{HO}$, the trajectory oscillates between two saddle-focus again. Subsequently, the trajectory returns to $\mathrm{QS}$ by $\mathrm{HB}_{3}$ again. Then, the pattern spiking $\mathrm{SP}_{3}$ is genetared by $\mathrm{HB}_{2}$. Although both are caused by $\mathrm{HB}$, the amplitude of oscillation of the third model is significantly smaller than that of the first two, and oscillates in the lower half of the equilibrium point. Sequentially, the system exits $\mathrm{SP}_{3}$ return to $\mathrm{QS}$ at $\mathrm{HB}_{1}$. Finally, the trajectory follows the equilibrium point to $\mathrm{BP}_{2}$ and returns to the first pattern of $\mathrm{BO}$. So far, the whole oscillation process has been analyzed completely. This type is also called delayedpitchfork/Hopf/homoclinic cascade induced quasi-periodic bursting .because it keeps switching between these bifurcations.

4.5 The quasi-periodic bursting caused by PBDIH-HO-HB/HB-HO-HB/HB-HO-HB-PBDIH cascade

To further explore the effect of parameter $b$, we choose $b=4$ to analyze the system dynamic.

\subsubsection{Bifurcation diagram and Lyapunov exponents analysis}

The bifurcation diagram and Lyapunov exponents with varying $g(t)$ can be acquired from system (2), and shown in Fig. 10. Interestingly, the local bifurcation mainly occurs in the region of $g(t)<0$, in which the BP and three pairs $\mathrm{HB}$ are located at $(-0.9713,0),(-0.9307, \pm 1.449),(-5789, \pm 1.449)$ and $(-0.1099, \pm 1.449)$ respectively. Besides, the results indicate that system (2) presents periodic attractors in most of the areas of $g(t)$, while chaotic attractors are only observed around HB. Eventually, Fig. 7 shows that the system behaves as a quasi-periodic bursting oscillation under the control of $g(t)$.

\subsubsection{Mechanism analysis}

The time series of $x$ and $g(t)$ are presented in Fig. 11(a) and 11(b). While the superposition graph for the evolution of equilibrium point and time series of $x$ and transformed phase diagram are plotted in Fig. 11(c) and 11(d). It is obvious that the oscillation process can be divided into three parts, which are represented by green, pink and purple trajectory respectively. Firstly, the pattern 1 is induced by cascaded PBDIHHO-HB. Unlike the others, the pattern 2 is induced by HB$\mathrm{HO}-\mathrm{HB}$, and the pattern 3 is induced by cascaded HB-HOHB-PBDIH. Therefore, such oscillation is also known as a quasi-periodic bursting caused by PBDIH-HO-HB/HB-HOHB/HB-HO-HB-PBDIH cascade. 

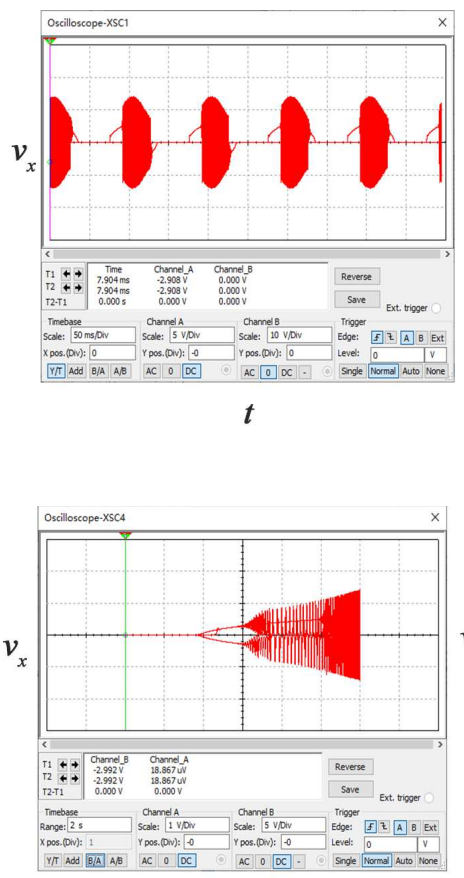

$g(t)$

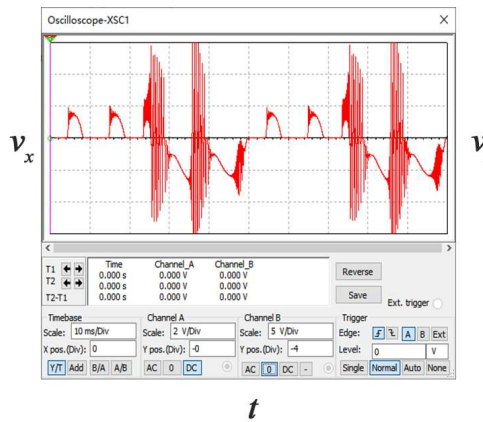

(a)

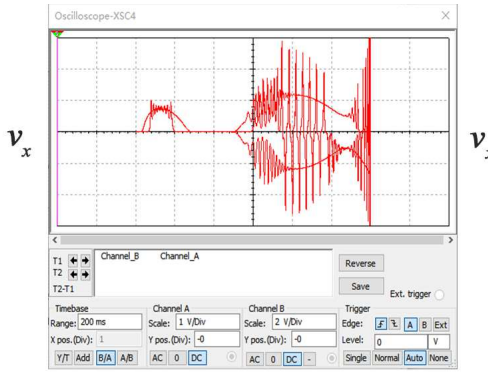

$g(t)$

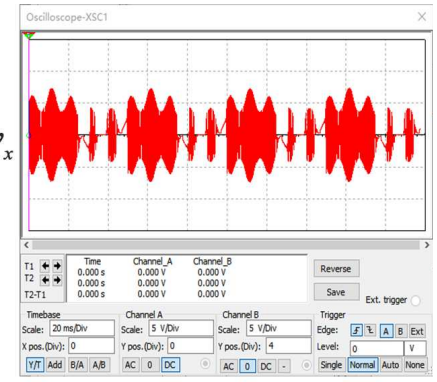

$t$

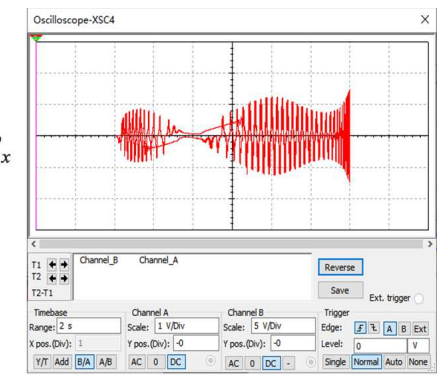

$g(t)$

(b)

Fig. 15 With $R_{13}=100 \mathrm{~K} \Omega$ and $\beta_{1}=\beta_{2}=3$, Multisim simulation results which time series and transformed phase diagram are shown for different parameters: (a) time series for case 1: $f_{1}=f_{2}=10 \mathrm{~Hz}$ and $R_{8}=50 \mathrm{~K} \Omega$, case $2: f_{1}=20 \mathrm{~Hz}, f_{2}=100 \mathrm{~Hz}$ and $R_{8}=50 \mathrm{~K} \Omega$, case 3 : $f_{1}=20 \mathrm{~Hz}, f_{2}=100 \mathrm{~Hz}, R_{8}=25 \mathrm{~K} \Omega$, (b) transformed phase diagram for case 1: $f_{1}=f_{2}=10 \mathrm{~Hz}$ and $R_{8}=50 \mathrm{~K} \Omega$, case $2: f_{1}=20 \mathrm{~Hz}, f_{2}=100 \mathrm{~Hz}$ and $R_{8}=50 \mathrm{~K} \Omega$, case 3: $f_{1}=20 \mathrm{~Hz}, f_{2}=100 \mathrm{~Hz}, R_{8}=25 \mathrm{~K} \Omega$.

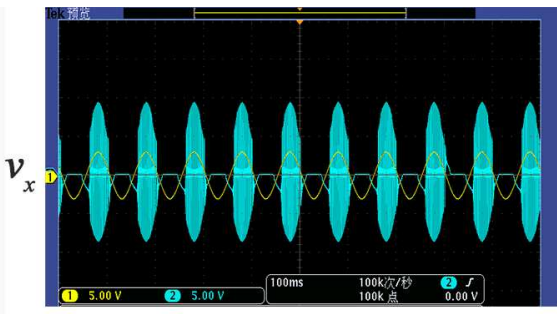

$t$

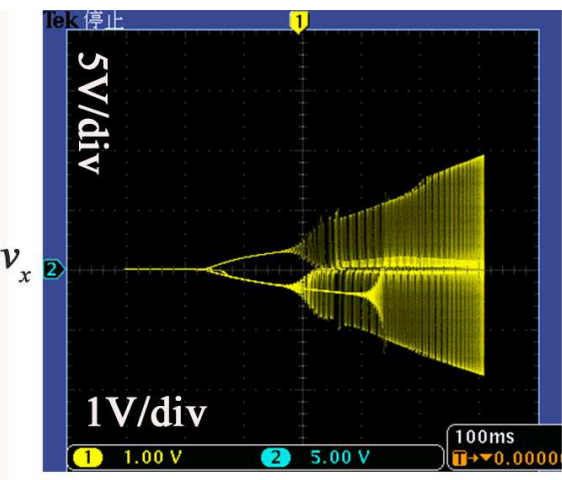

$g(t)$

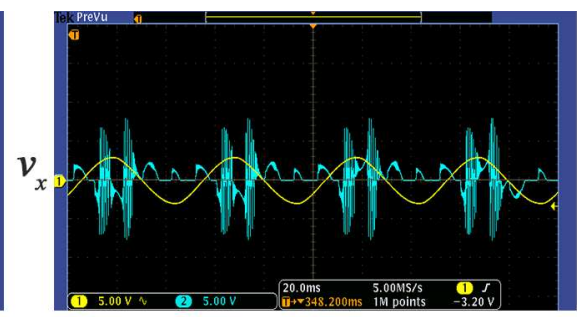

$\boldsymbol{t}$

(a)

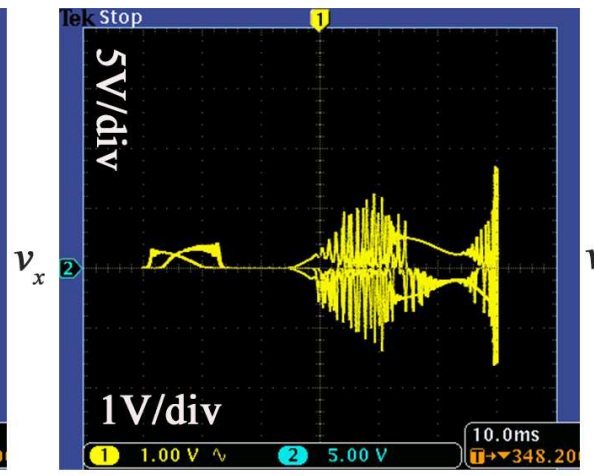

$g(t)$

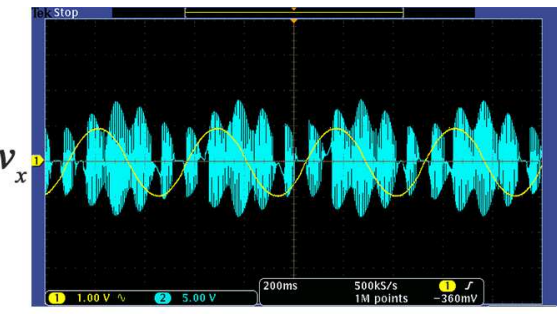

$t$

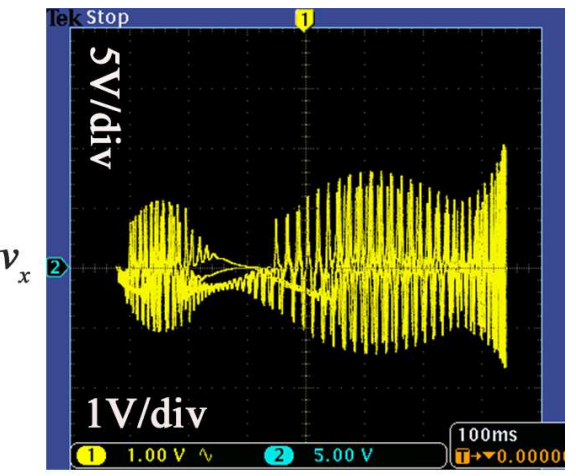

$g(t)$

(b)

Fig. 16 Complex dynamics for varing-parameter are observed from oscilloscope (the detailed parameters correspond to Fig. 15): (a) time series of $x$, (b) transformed phase diagrams. 
4.6 The second delayed-pitchfork/Hopf/homoclinic cascade induced chaotic bursting

When $b$ increases to 5 , a novel class of chaotic bursting can be observed from system (1).

\subsubsection{Bifurcation diagram and Lyapunov exponents analysis}

The bifurcation diagram and Lyapunov exponents of generalized slow variable $g(t)$ are shown in Fig. 12. From which, we can konw that the path for system (1) to enter the chaotic state is HB, FPDB. The system exhibits chaos in most regions with slow-varying parameter. Besides, several period windows are generated by system (2). At the same time, Fig. 7(b) shows that the maximum Lyapunov exponent LE1 = 0.2154 , which means that the motion is chaotic.

\subsubsection{Mechanism analysis}

The time series of $x$ are plotted in Fig. 13(a) and 13(b). There are two kinds of spiking transitions in a SP, including periodic spiking states (PSP) in the region II and IV, and chaotic spiking states (CSP) in the region I, III and V. The superposition graph for the evolution of equilibrium point and time series of $x$, and transformed phase diagram are presented in Fig. 13(c) and 13(d) separately. Firstly, when the trajectory slowly passes through the BP point, the system (1) enters the CSP through PBDIH. Then, the trajectory reaches the $\mathrm{HB} 2 \pm$ point, which generates PSP from the upper and lower branches of the equilibrium. Next, the trajectory exits the PSP and entering CSP again by $\mathrm{HB}_{ \pm}$point. Finally, the trajectory returns to QS by BP point again. Therefore, the oscillation process can be understood as a chaotic bursting caused by delayed-pitchfork/Hopf/homoclinic cascade.

\section{Circuit design and hardware experiment}

In this section, the above dynamics are realized and verified by circuit simulation and hardware experiment. The simulation is completed by the Multisim 13.0 circuit simulation platform, and the hardware experiment is conducted on the breadboard.

To begin with, system (1) can be normalized after differentia integral transformation, as follows,

$$
\left\{\begin{array}{l}
x=\tau_{0} \int-a x-a(-y) d t \\
y=\tau_{0} \int-w_{1}(-x)-x z-b(-y) d t \\
z=\tau_{0} \int-x(-x)-c z-w_{2} d t
\end{array}\right.
$$

where $\tau_{0}$ is a time constant determined by integral parameter setting in the circuit. To increase the frequency of the excitation in analog circuit, $\tau_{0}$ is set to 10000 .
Table 3 The circuit parameters

\begin{tabular}{ccc}
\hline Parameters & Significations & Value \\
\hline$R_{0}, R_{1}, R_{2}, R_{4}, R_{5}, R_{6}$ & Resistance & $10 \mathrm{k} \Omega$ \\
$R_{7}, R_{10}, R_{11}, R_{12}, R_{16}, R_{17}$ & Resistance & $10 \mathrm{k} \Omega$ \\
$R_{3}, R_{9}, R_{14}, R_{15}$ & Resistance & $100 \mathrm{k} \Omega$ \\
$R_{8}, R_{13}$ & Resistance & Variable \\
$C_{0}$ & Capacitance & $10 \mathrm{nF}$ \\
$w_{1}$ & Excitation source & Variable \\
$w_{2}$ & Excitation source & Variable \\
\hline \hline
\end{tabular}

Based on (23), an analog circuit is designed to realize the circuit system by using integrated circuits and discrete components. The circuit diagram and the experiment equipment are shown in Fig. 14. Multiple UA741, AD633 are used to realize integration, multiplication and addition of $\mathrm{V} x, \mathrm{~V} y$ and $\mathrm{V} z$. Besides, $w_{1}$ and $w_{2}$ are two sine signals genarated by signal genarator (YB1615P).

In order to obtain the specific component parameters, based on Fig. (14)(a), the circuit expression also should be deduced as follows

$$
\left\{\begin{array}{l}
v_{x}=\frac{1}{R_{0} C_{0}} \int-\frac{R_{3}}{R_{1}} v_{x}-\frac{R_{3}}{R_{2}}\left(-v_{y}\right) d t \\
v_{y}=\frac{1}{R_{0} C_{0}} \int-\frac{R_{10}}{R_{7}} w_{1}\left(-v_{x}\right)-\frac{R_{10}}{R_{8}} v_{x} v_{z}-\frac{R_{10}}{R_{9}}\left(-v_{y}\right) d t \\
v_{z}=\frac{1}{R_{0} C_{0}} \int-\frac{R_{17}}{R_{14}} v_{x}\left(-v_{x}\right)-\frac{R_{17}}{R_{15}} v_{z}-\frac{R_{17}}{R_{16}} w_{2} d t
\end{array}\right.
$$

Comparing (23) and (24), and setting the circuit parameters as those in Table 3. Finally, the results of the simulation are shown in Fig. 15, and the corresponding hardware experimental results are observed by an oscilloscope (Tektronix MSO3032) and shown in Fig. 16. Consequantly, all of experiment results are consistent with the numerical analysis and simulation results.

\section{Conclusion}

In this work, we study a class of multi-timescale non-autonomous dynamical systems with parametric and external excitation. Theoretical analysis and numerical simulation are carried alout to comprehensively analyze the characteristics and dynamic behavior of the system. Besides, the characteristics of equilibrium and local bifurcation are comprehensively analyzed. Interestingly, various kinds of multi-bifurcation cascaded bursting oscillations are observed by changing the frequency of two excitations and related system parameters. In conclusion, the system and excitation parameter play a crucial role in the formation of dynaimcs. By using excitation transformation method and fast-slow analysis method, their formation mechanism is analyzed in detail. Furthermore, a 
hardware analog circuit is designed to verify these complex bursting oscillations, and circuit simulation and breadboard experiments have been conducted in this study. This work is of great practical significance, which not only helps to enrich the existing theory, but also provides a physically realizable circuit for the application of bursting oscillation signal.

Acknowledgements This work was supported by the Research Foundation of Education Department of Hunan Province, China(Grant No. 20B567), the Natural Science Foundation of Hunan Province, China(Grant No. 2019JJ50624), and National Natural Science Foundation of China (Grant No. 62071411). This work was also funded by the China Scholarship Council (Grant No. 201808430258).

\section{Conflict of interest}

The authors declare that we have no conflicts of interests about the publication of this paper.

\section{References}

1. D. Matzakos-Karvouniari, L. Gil, E. Orendorff, O. Marre, S. Picaud, and B. Cessac, A biophysical model explains the spontaneous bursting behavior in the developing retina, Sci. Rep., 9, 1859 (2019)

2. M. I. Español and H. G. Rotstein, Complex mixed-mode oscillatory patterns in a periodically forced excitable belousov-zhabotinsky reaction model, Chaos, 25, 064612 (2015)

3. Y. Chen, C. K. Tse, S. Qiu, L. Lindenmuller, and W. Schwarz, Coexisting fast-scale and slow-scale instability in current-mode controlled dc/dc converters: Analysis, simulation and experimental results, IEEE Trans. Circuits Syst. I-Regul. Pap., 55, 3335-3348 (2008)

4. H. Li, D. Chen, X. Gao, X. Wang, Q. Han, and C. Wu, Fast-slow dynamics of a hydropower generation system with multi-time scales, Mech. Syst. Signal Proc., 110, 458 - 468 (2018)

5. E. N. Lorenz, Deterministic Nonperiodic Flow, J. Atmos. Sci, 20, 130-141 (1963)

6. S. Yu, J. Lu, X. Yu, and G. Chen, Design and implementation of grid multiwing hyperchaotic lorenz system family via switching control and constructing super-heteroclinic loops, IEEE Trans. Circuits Syst. I-Regul. Pap., 59, 1015-1028 (2012)

7. K. Cho and T. Miyano, Chaotic cryptography using augmented lorenz equations aided by quantum key distribution, IEEE Trans. Circuits Syst. I-Regul. Pap., 62, 478-487 (2015)

8. S. Cang, Y. Li, R. Zhang and Z. Wang, Hidden and self-excited coexisting attractors in a Lorenz-like system with two equilibrium points, Nonlinear Dyn., 95, 381-390 (2019)

9. E. M. Izhikevich, Neural excitability, spiking and bursting, Int. J. Bifurcation Chaos, 10, 1171-1266 (2000)

10. R. T. Siewe, U. S. Domguia, and P. Woafo, Generation of pulselike and bursting-like oscillations from nonlinear systems using embedded technologies and applications to excite mechanical arms, Commun. Nonlinear Sci. Numer. Simul., 69, 343-359 (2019)

11. Q. Duan, Z. Jing, X. Zou, Y. Wang, K. Yang, T. Zhang, S. Wu, R. Huang, and Y. Yang, Spiking neurons with spatiotemporal dynamics and gain modulation for monolithically integrated memristive neural networks, Nat. Commun., 11, 3399 (2020)

12. X. Huang, X. Ruan, F. Du, F. Liu, and L. Zhang, A pulsed power supply adopting active capacitor converter for low-voltage and lowfrequency pulsed loads, IEEE Trans. Power Electron., 33, 9219-9230 (2018)
13. Q. Bi, R. Ma, and Z. Zhang, Bifurcation mechanism of the bursting oscillations in periodically excited dynamical system with two time scales, Nonlinear Dyn., 79, 101-110 (2015)

14. Q. Bi and Z. Zhang, Bursting phenomena as well as the bifurcation mechanism in controlled lorenz oscillator with two time scales, Phys. Lett. A, 375, 1183-1190 (2011)

15. H. Bao, A. Hu, W. Liu, and B. Bao, Hidden bursting firings and bifurcation mechanisms in memristive neuron model with threshold electromagnetic induction, IEEE Trans. Neural Netw. Learn. Syst., 31, 502-511 (2020)

16. X. Han, B. Jiang, and Q. Bi, 3-torus, quasi-periodic bursting, symmetric subHopf/fold-cycle bursting, subHopf/fold-cycle bursting and their relation, Nonlinear Dyn., 61, 667-676 (2010)

17. X. Han, Y. Zhang, Q. Bi, and J. Kurths, Two novel bursting patterns in the duffing system with multiple-frequency slow parametric excitations, Chaos, 28, 043111 (2018)

18. X. Han, Q. Bi, P. Ji, and J. Kurths, Fast-slow analysis for parametrically and externally excited systems with two slow rationally related excitation frequencies, Phys. Rev. E, 92, 012911 (2015)

19. R. Bertram and J. E. Rubin, Multi-timescale systems and fast-slow analysis, Math. Biosci., 287, 105-121 (2017)

20. X. Han, M. Wei, Q. Bi, and J. Kurths, Obtaining amplitudemodulated bursting by multiple-frequency slow parametric modulation, Phys. Rev. E, 97, 012202 (2018)

21. G. Cai, Z. Tan, W. Zhou, and W. Tu, Dynamical analysis of a new chaotic system and its chaotic control, Acta Phys. Sin., 56, 6230-6237 (2007)

22. M. Wang, Y. Zeng, G. Chen, and J. He, Nonresonant parametric control of chen's system, Acta Phys. Sin., 60, 010509 (2011)

23. E.-H. Park, M. A. Zaks, and J. Kurths, Phase synchronization in the forced lorenz system, Phys. Rev. E, 60, 6627-6638 (1999)

24. W. Teka, J. Tabak, and R. Bertram, The relationship between two fast/slow analysis techniques for bursting oscillations, Chaos, 22, 043117 (2012)

25. X. Han, Y. Liu, Q. Bi, and J. Kurths, Frequency-truncation fastslow analysis for parametrically and externally excited systems with two slow incommensurate excitation frequencies, Commun. Nonlinear Sci. Numer. Simul., 72, 16 - 25 (2019)

26. E. Cho, De moivre's formula for quaternions, Appl. Math. Lett., $11,33-35$ (1998)

27. S. Yu, J. Lu, and G. Chen, Theoretical design and circuit implementation of multidirectional multi-torus chaotic attractors, IEEE Trans. Circuits Syst. I-Regul. Pap., 54, 2087-2098 (2007)

28. C. Zhou, Z. Li, F. Xie, M. Ma and Y. Zhang, Bursting oscillations in Sprott B system with multi-frequency slow excitations: two novel "Hopf/Hopf"-hysteresis-induced bursting and complex AMB rhythms, Nonlinear Dyn., 97, 2799-2811 (2019)

29. E. X. DeJesus and C. Kaufman, Routh-hurwitz criterion in the examination of eigenvalues of a system of nonlinear ordinary differential equations, Phys. Rev. A, 35, 5288-5290 (1987)

30. S. Sancho, A. Suarez, and P. S. Roman, Floquet analysis of the intermittence route to chaos through a pitchfork bifurcation, IEEE Trans. Circuits Syst. I-Fundam. Theor. Appl., 48, 374-377 (2001)

31. G. Revel, A. E. Leon, D. M. Alonso, and J. L. Moiola, Bifurcation analysis on a multimachine power system model, IEEE Trans. Circuits Syst. I-Regul. Pap., 57, 937-949 (2010) 


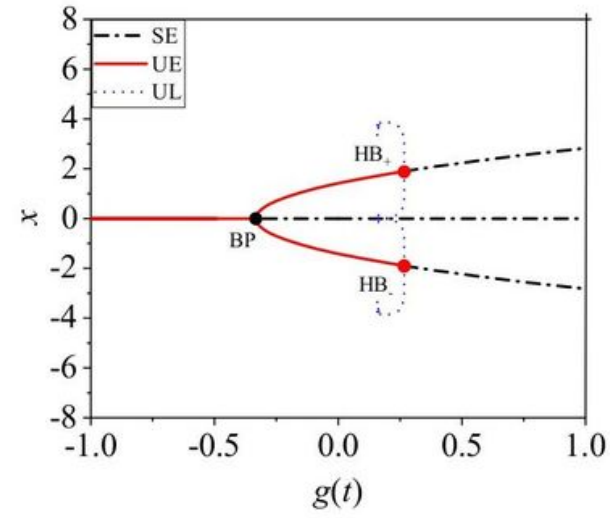

(a)

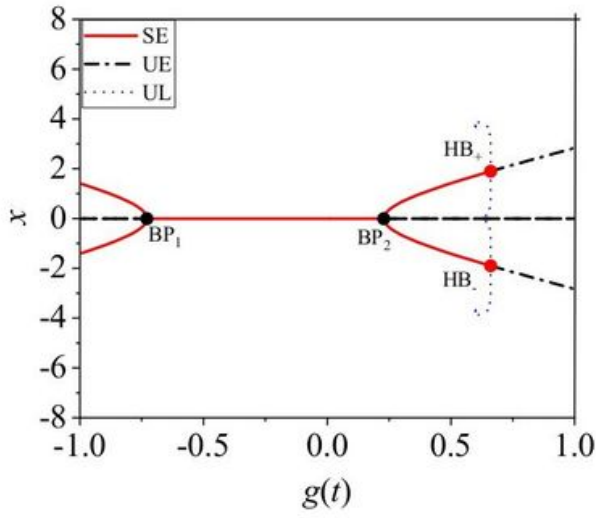

(b)

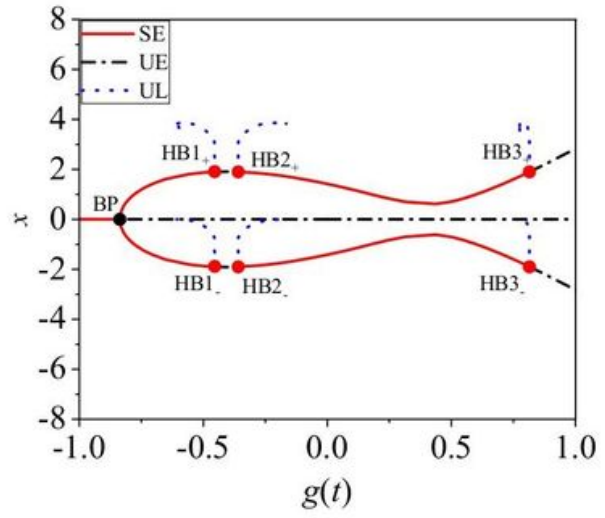

(c)

\section{Figure 1}

With $b=2, f 2=0.001$ and varing $f 1$, local bifurcation diagram are present: (a) $f 1=0.001$, (b) $f 1=0.002$, (c) $f 1=0.003$.

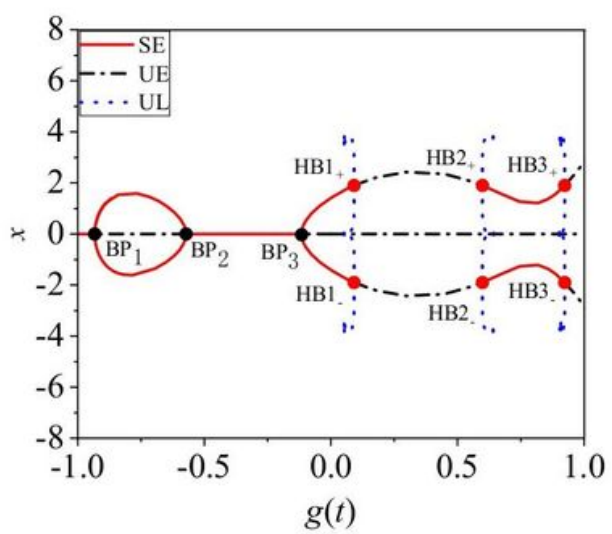

(a)

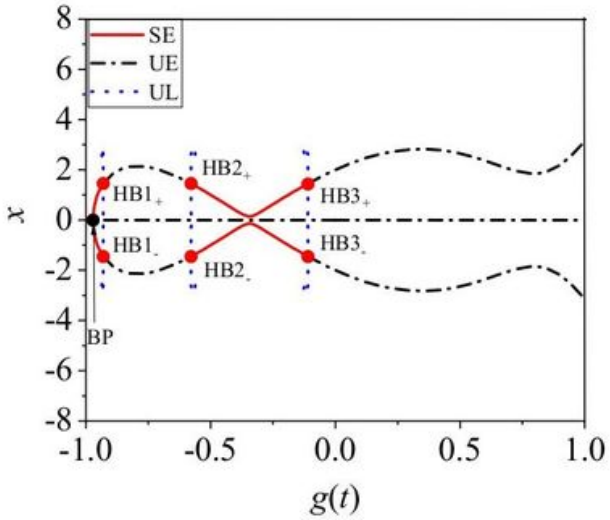

(b)

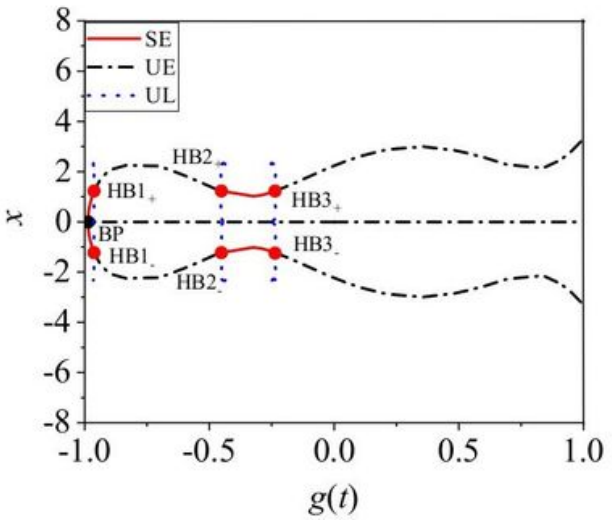

(c)

\section{Figure 2}

With $f 1=0.002, f 2=0.01$ and varing $b$, local bifurcation diagram are present: $(a) b=2,(b) b=4,(c) b=5$.

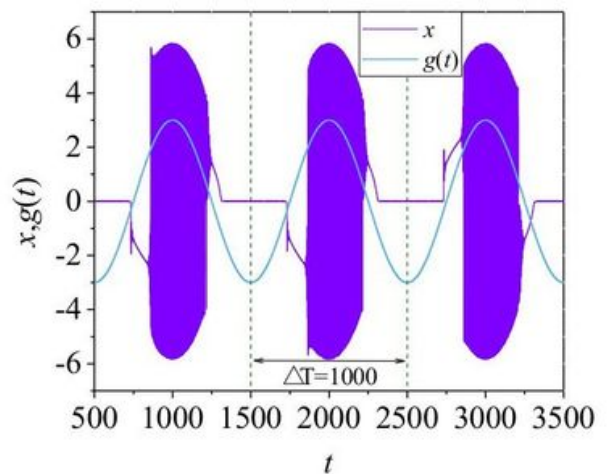

(a)

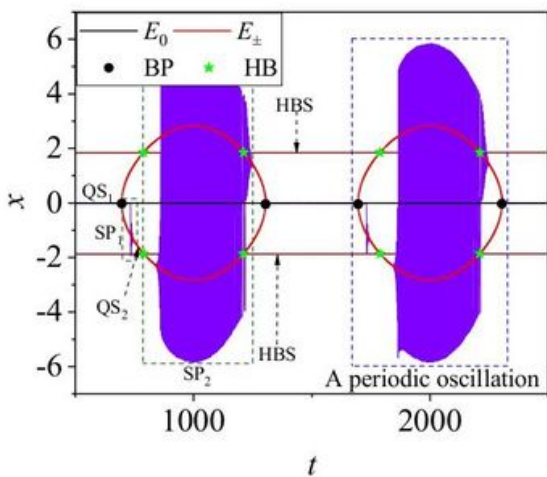

(b)

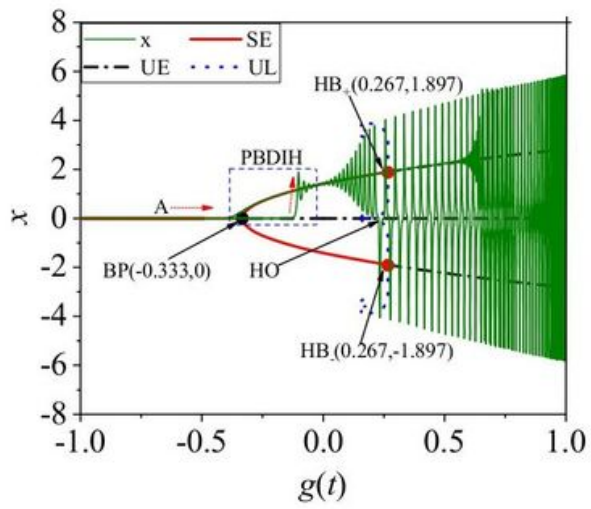

(c) 


\section{Figure 3}

Fast-slow dynamic for $\mathrm{f} 1 \mathrm{=} \mathrm{f} 2=0.001$ : (a) time series of $\mathrm{x}$, (b) The superposition graph for the evolution of equilibrium point and time series of $x$ along with time $t$, (c) transformed phase diagram.

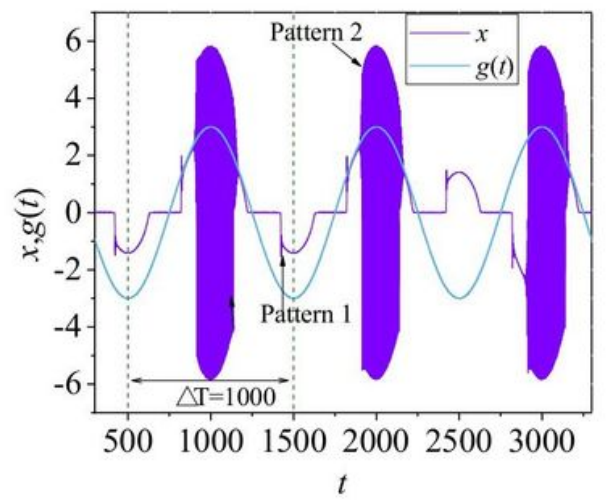

(a)

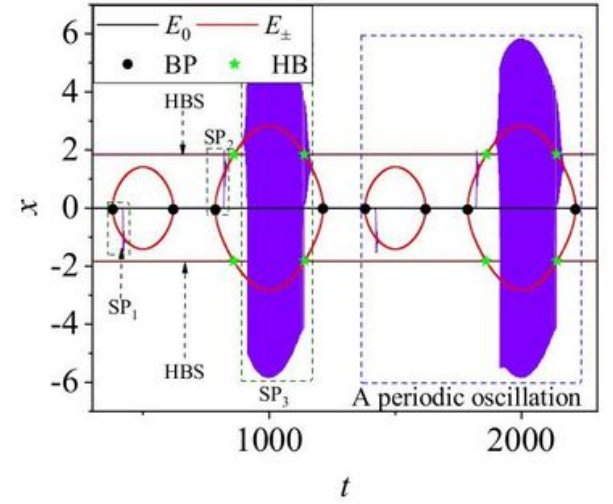

(b)

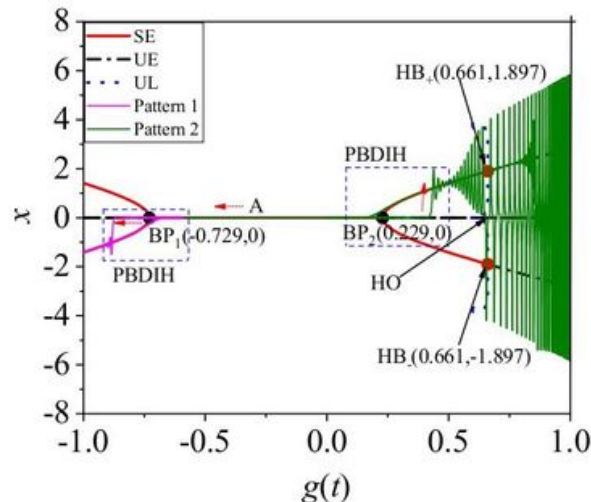

(c)

\section{Figure 4}

Fast-slow dynamic for $\mathrm{f} 1=0.002, \mathrm{f} 2=0.001$ : (a) time series of $\mathrm{x}$, (b) The superposition graph for the evolution of equilibrium point and time series of $x$ along with time $t$, (c) transformed phase diagram.

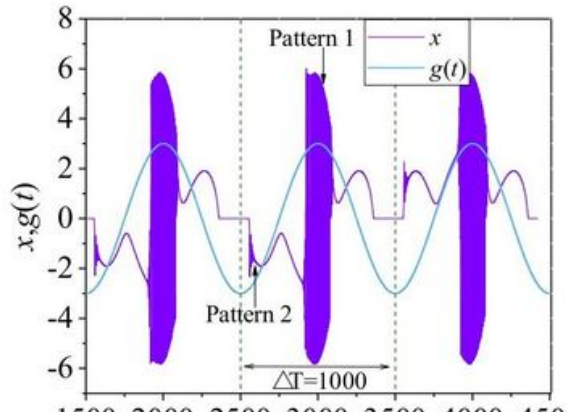

1500200025003000350040004500

(a)

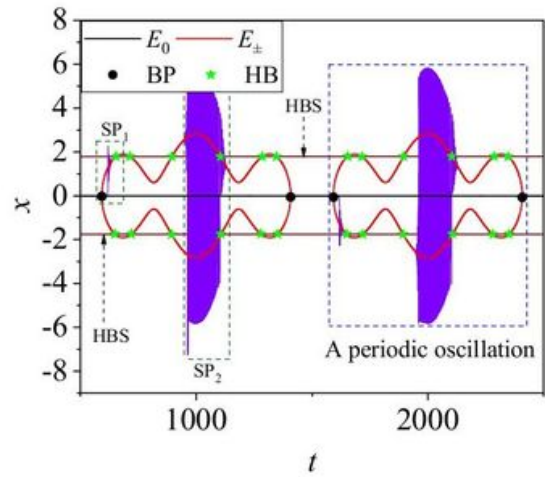

(b)

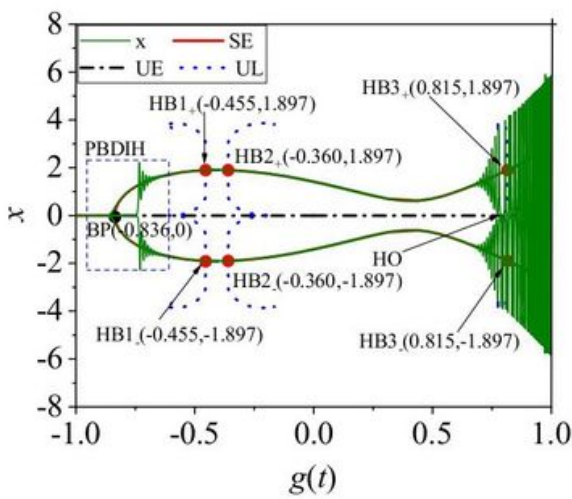

(c)

\section{Figure 5}

Fast-slow dynamic for $\mathrm{f} 1=0.003, \mathrm{f} 2=0.001$ : (a) time series of $\mathrm{x}$, (b) The superposition graph for the evolution of equilibrium point and time series of $x$ along with time $t,(c)$ transformed phase diagram. 


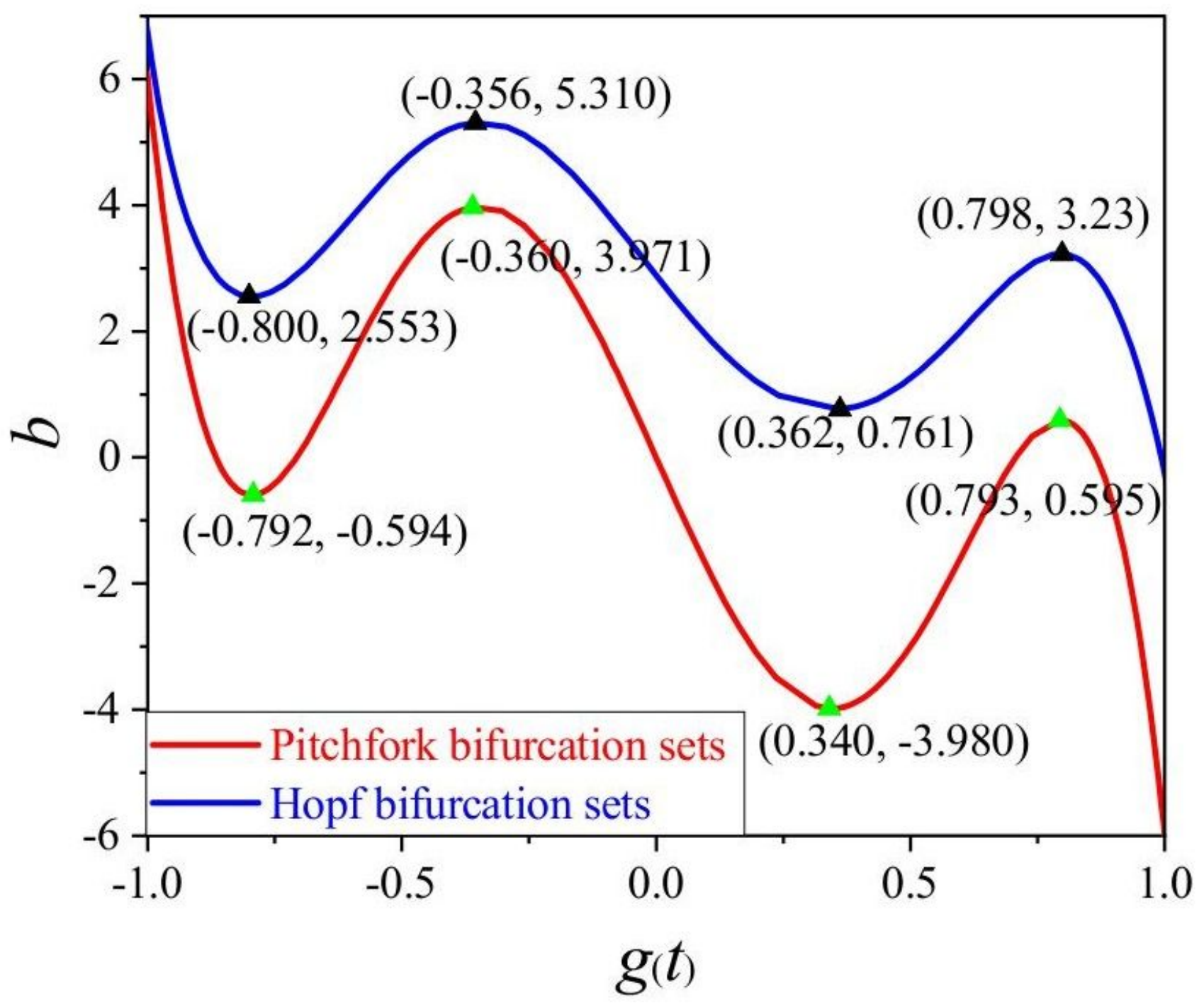

Figure 6

The two-parameter bifurcation sets of $b$ and $g(t)$ for $a=10, c=1, f 1=0.002$ and $f 2=0.01$. 


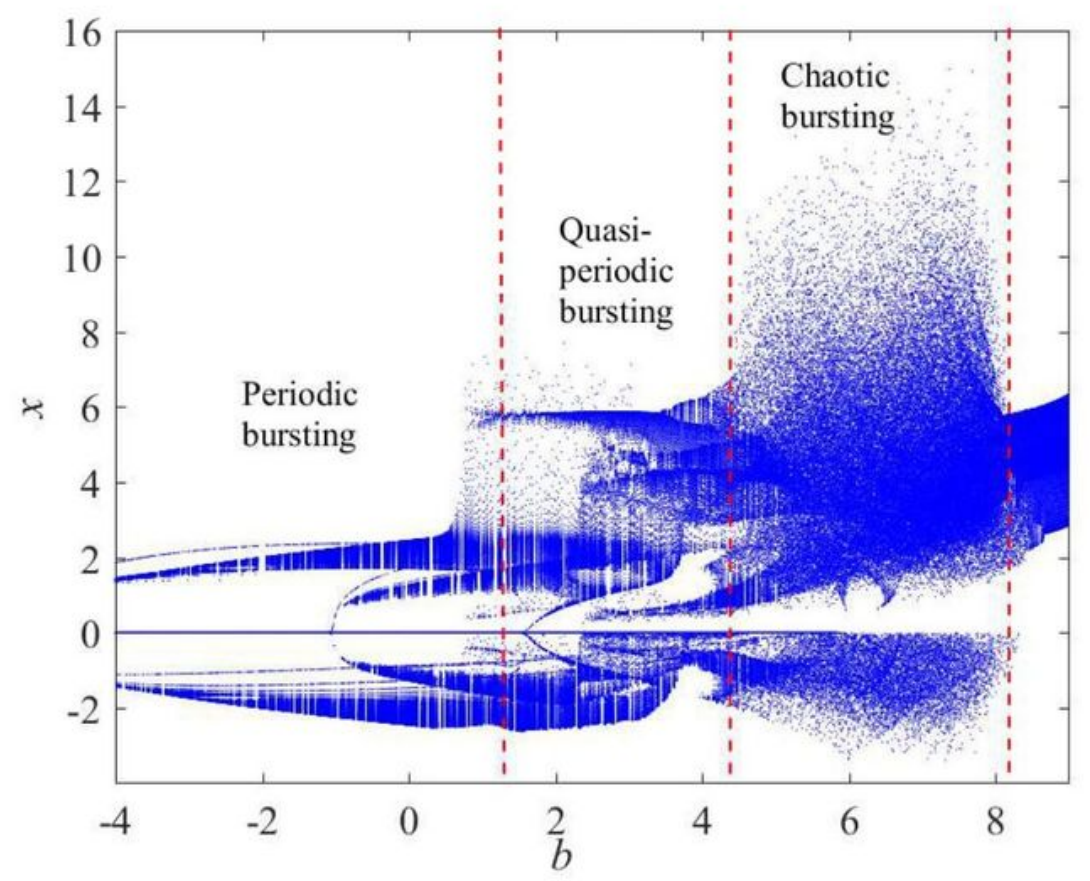

(a)

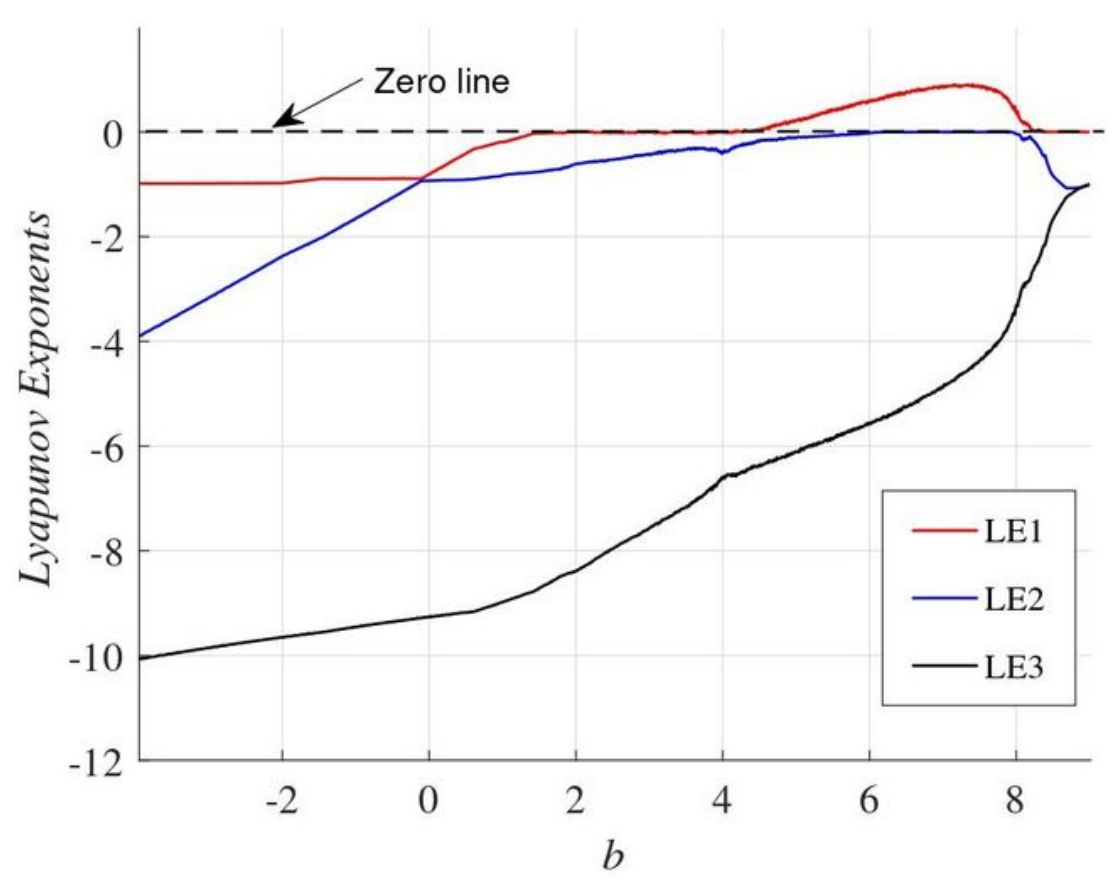

(b)

\section{Figure 7}

With $\mathrm{f} 1=0.002$ and $\mathrm{f} 2=0.01$, the dynamics of system ( 1 ) with b varying: (a) bifurcation diagram, (b) Lyapunov exponents. 


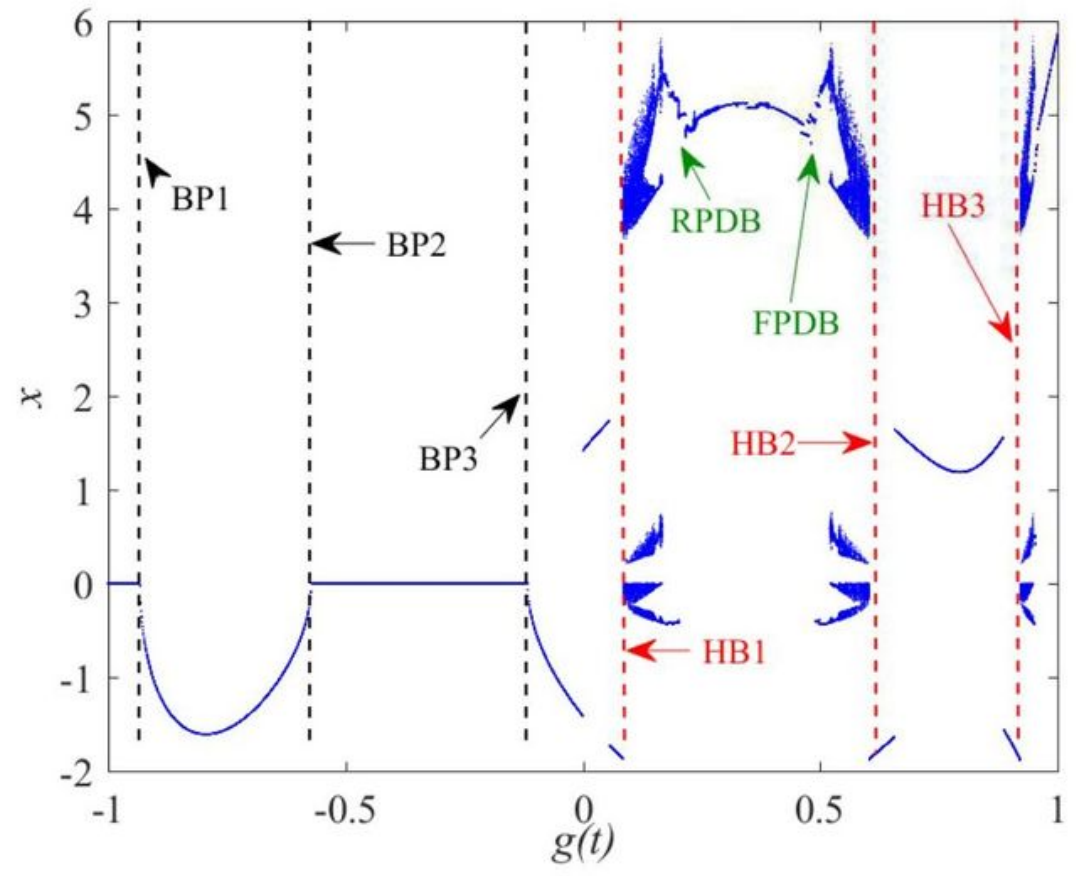

(a)

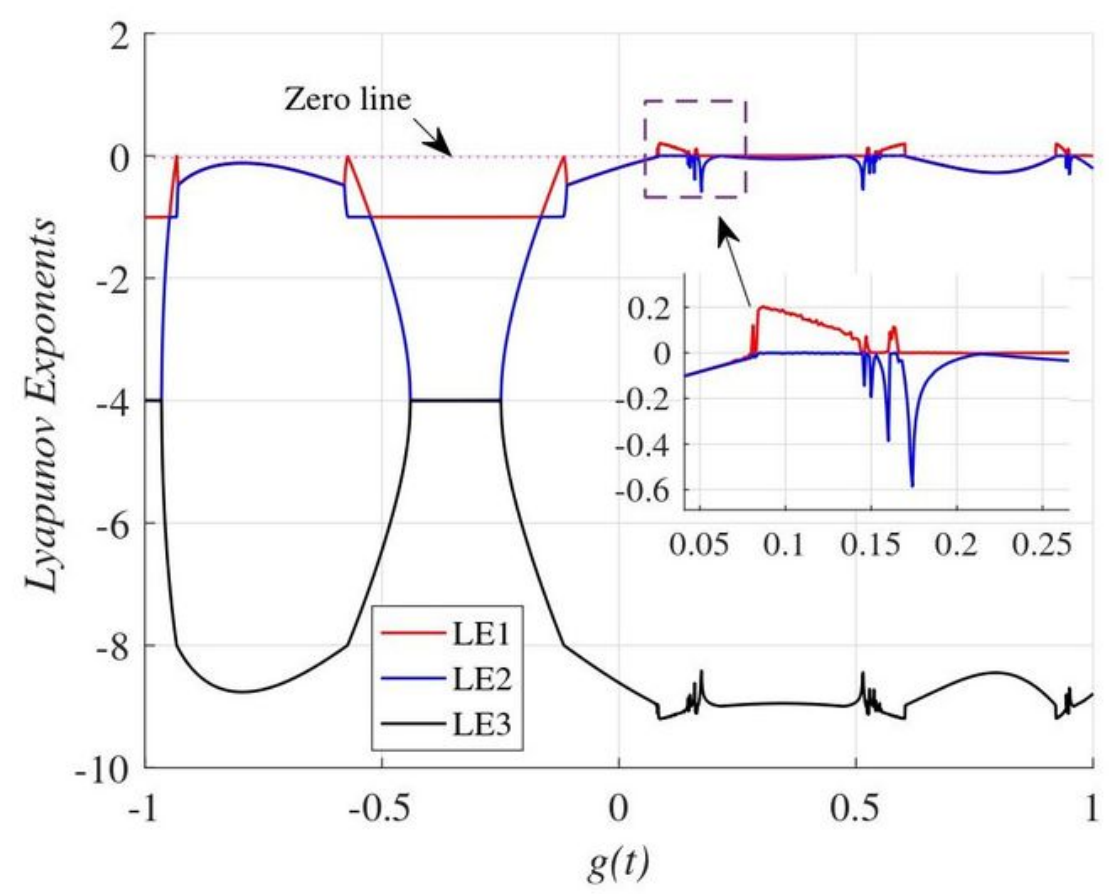

(b)

\section{Figure 8}

With $b=2, f 1=0.002$ and $f 2=0.01$, the dynamics of system ( 1 ) with $g(t)$ varying: (a) bifurcation diagram, (b) Lyapunov exponents. 


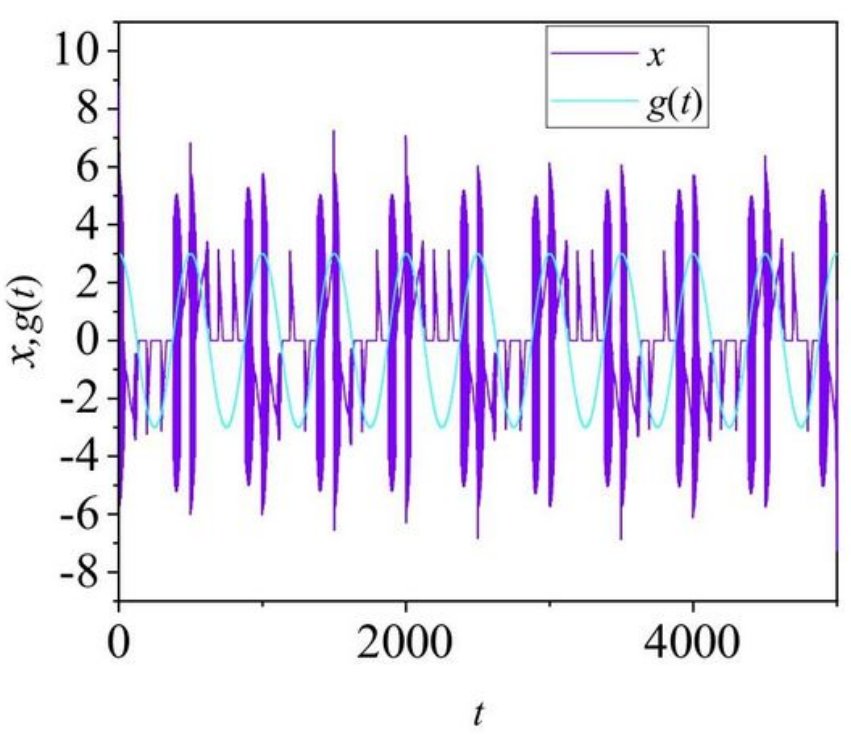

(a)

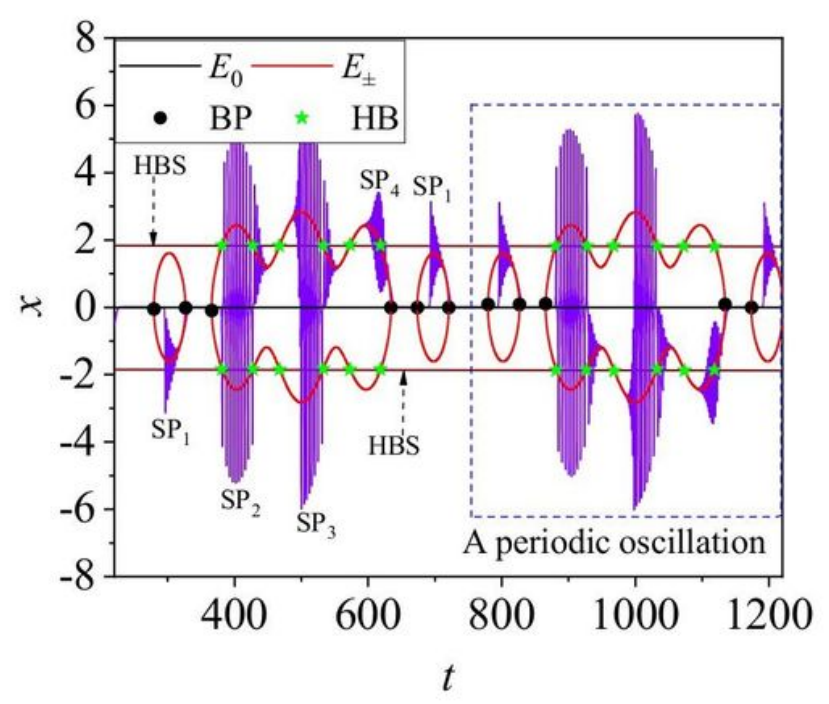

(c)

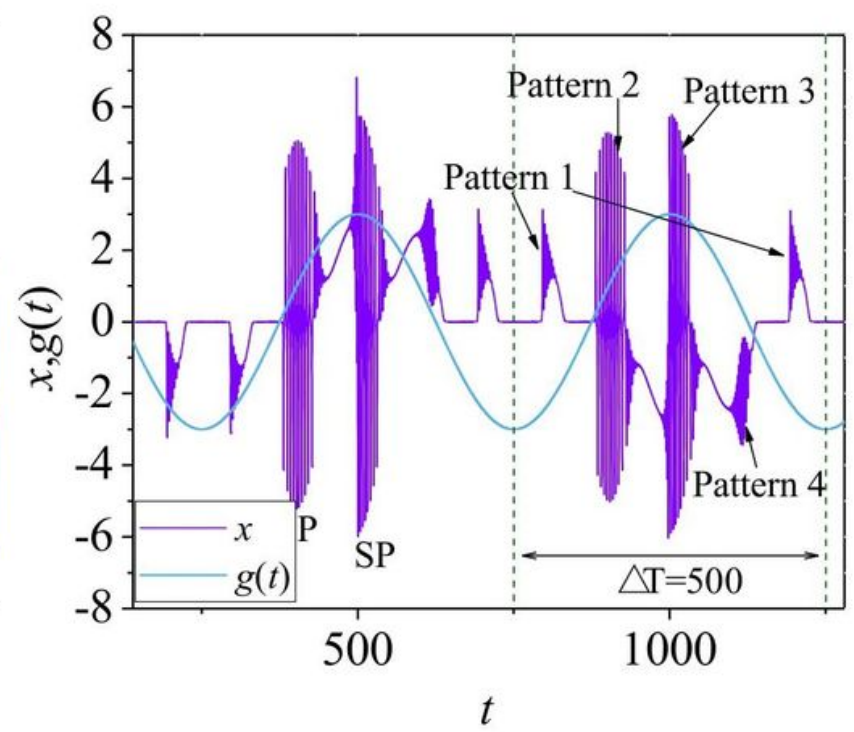

(b)

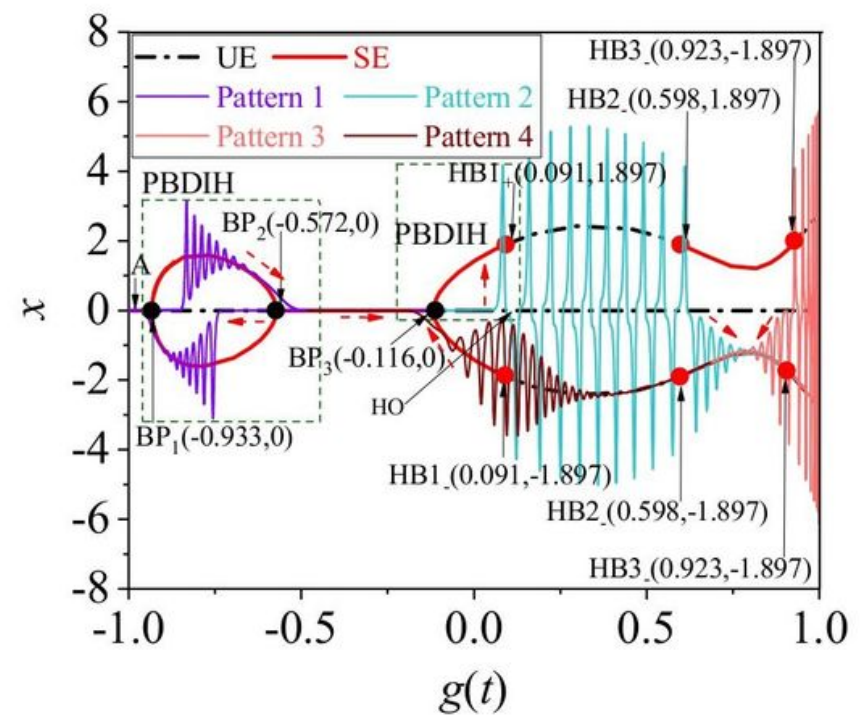

(d)

\section{Figure 9}

Fast-slow dynamic for $b=2$ : (a) whole time series of $x$ and $g(t),(b)$ Local enlarged time series of $x$ and $g(t)$, (c) The superposition graph for the evolution of equilibrium point and time series of $x$ along with time $t$, (d) transformed phase diagram. 


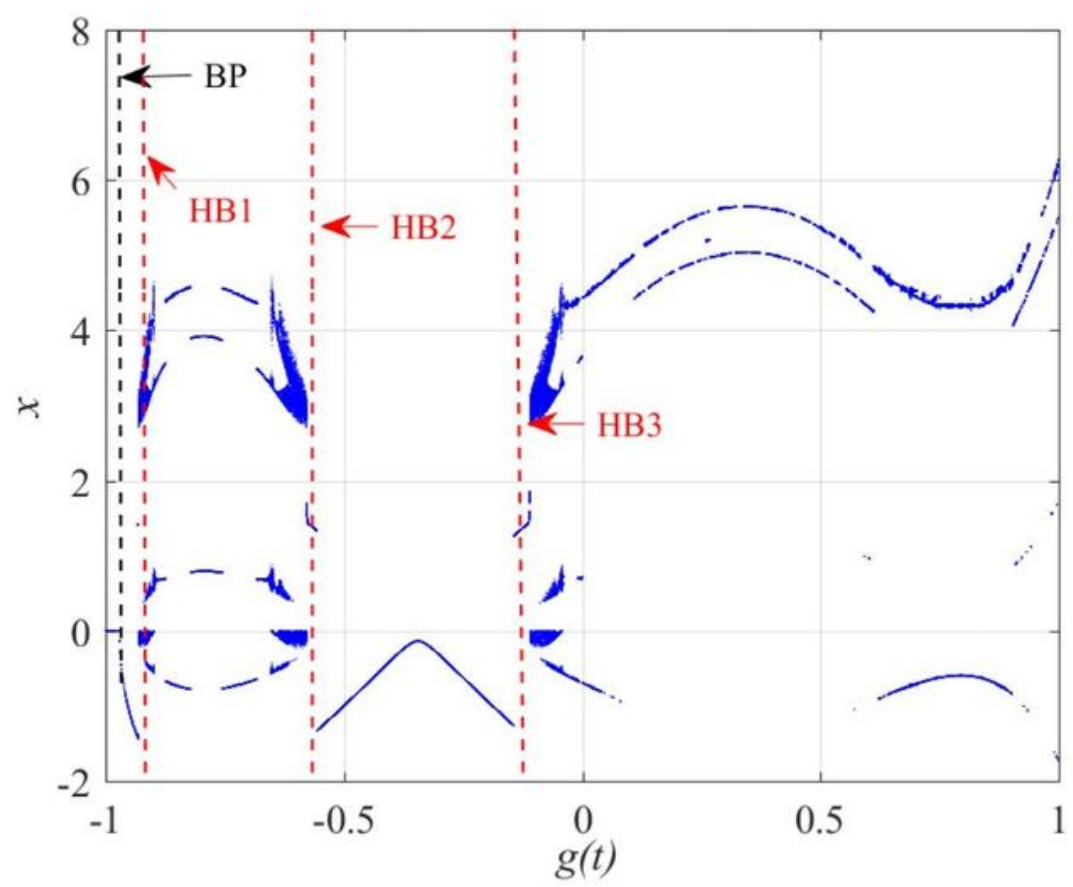

(a)

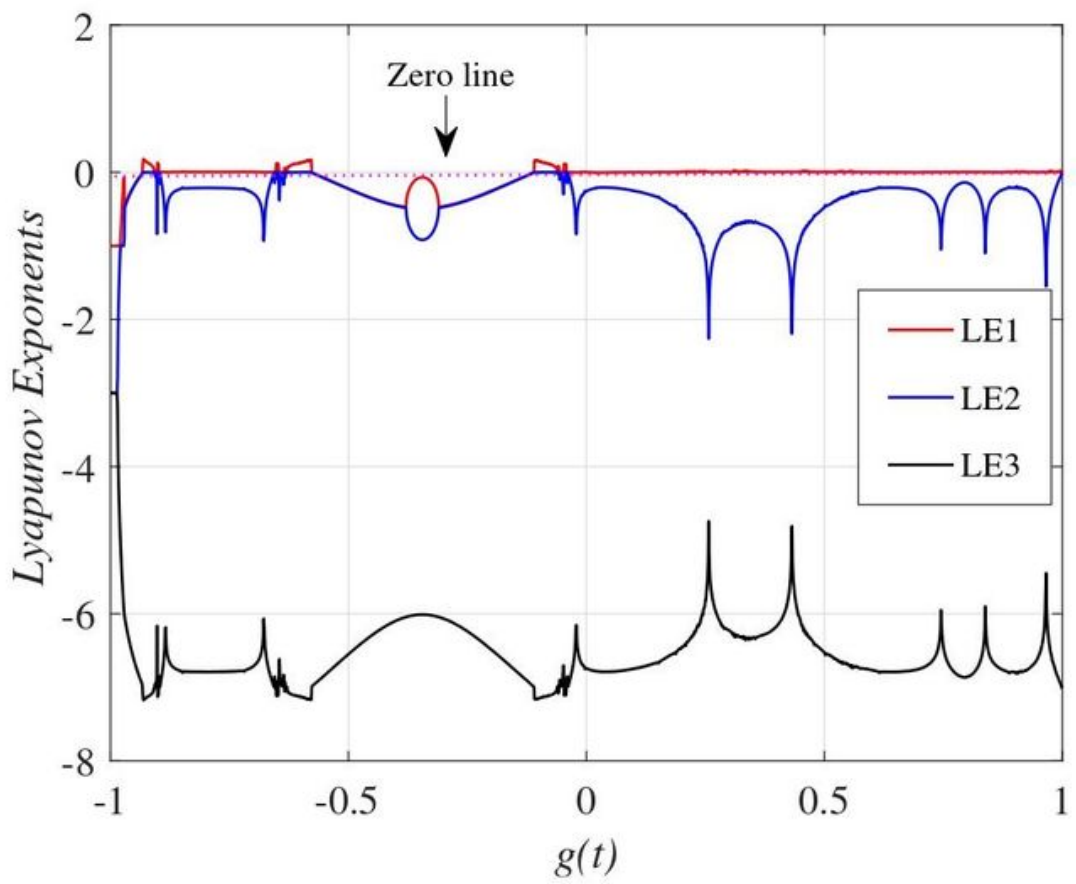

(b)

Figure 10

With $b=4, f 1=0.002$ and $f 2=0.01$, the dynamics of system ( 1 ) with $g(t)$ varying: (a) bifurcation diagram, (b) Lyapunov exponents. 


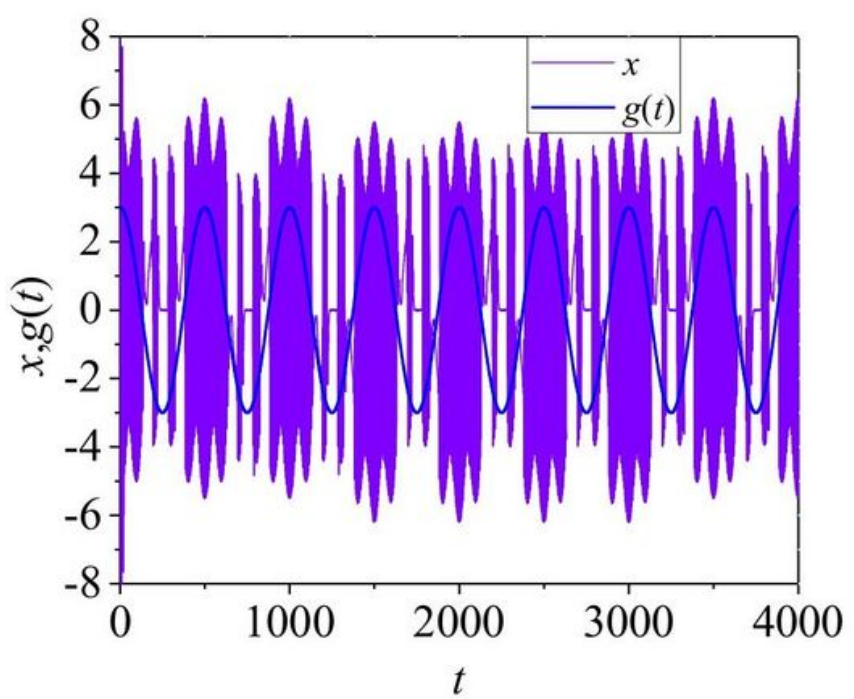

(a)

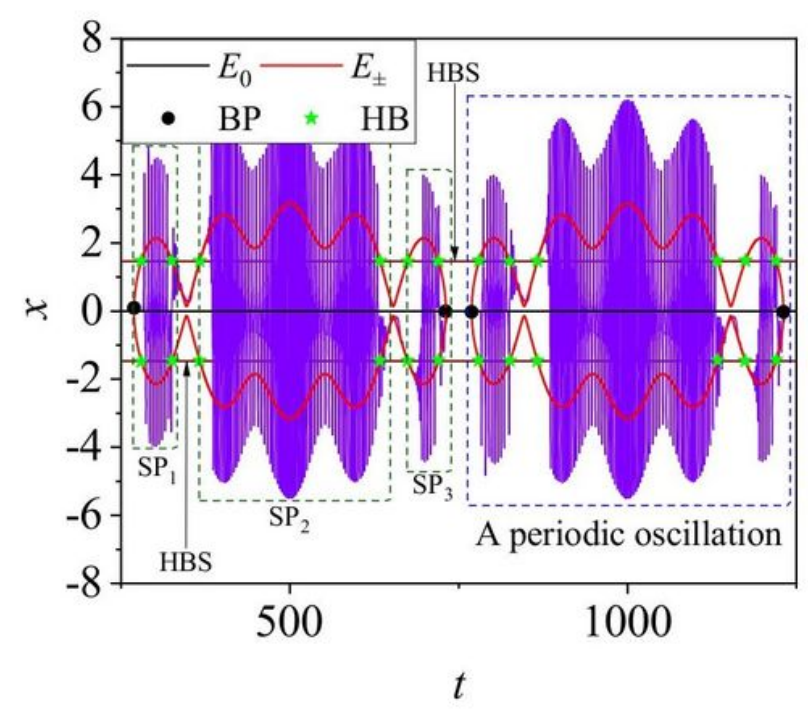

(c)

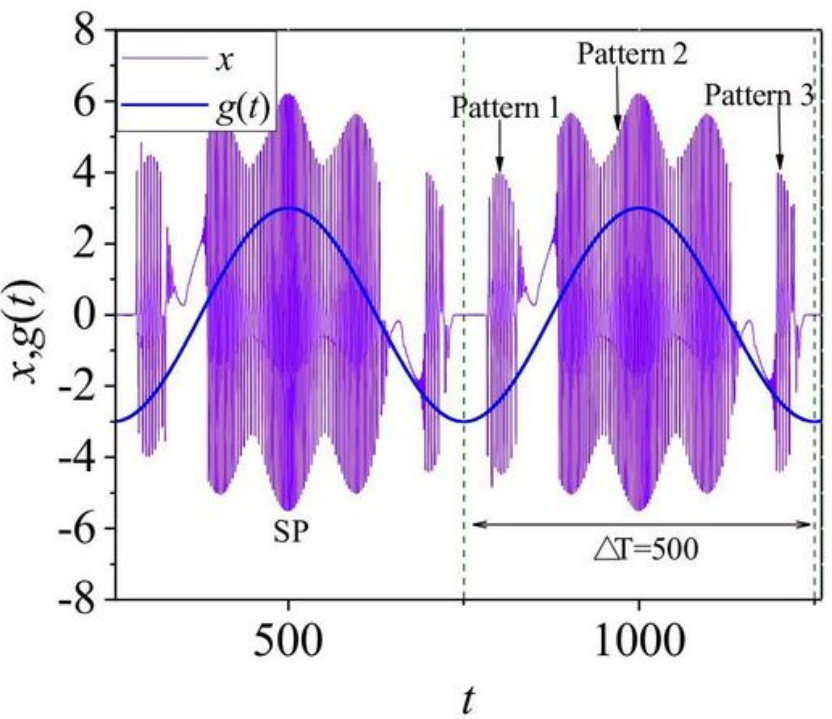

(b)

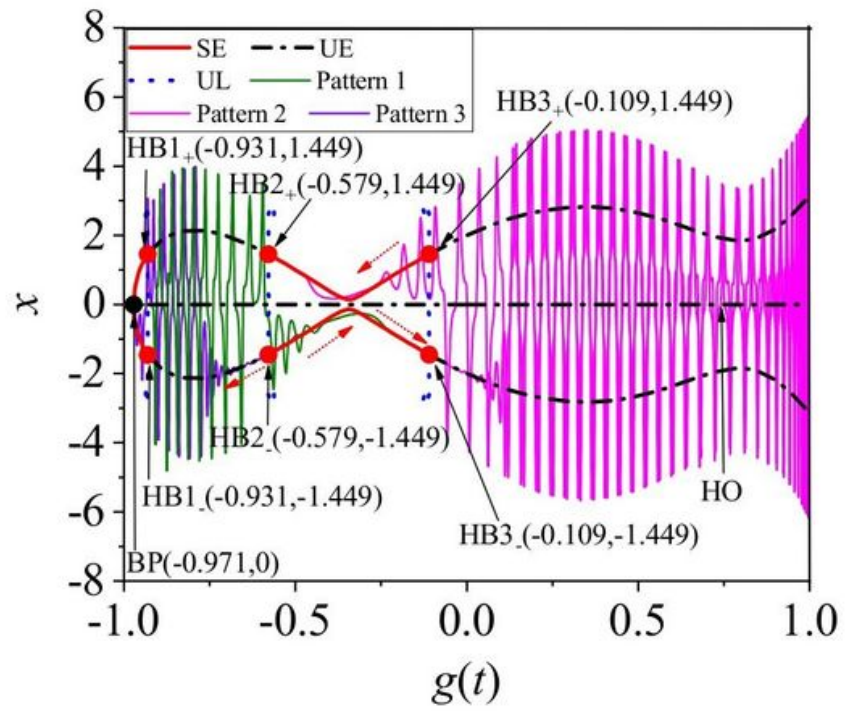

(d)

\section{Figure 11}

Fast-slow dynamic for $b=4$ : (a) whole time series of $x$ and $g(t)$, (b) Local enlarged time series of $x$ and $g(t)$, (c) The superposition graph for the evolution of equilibrium point and time series of $x$ along with time $t$, (d) transformed phase diagram. 


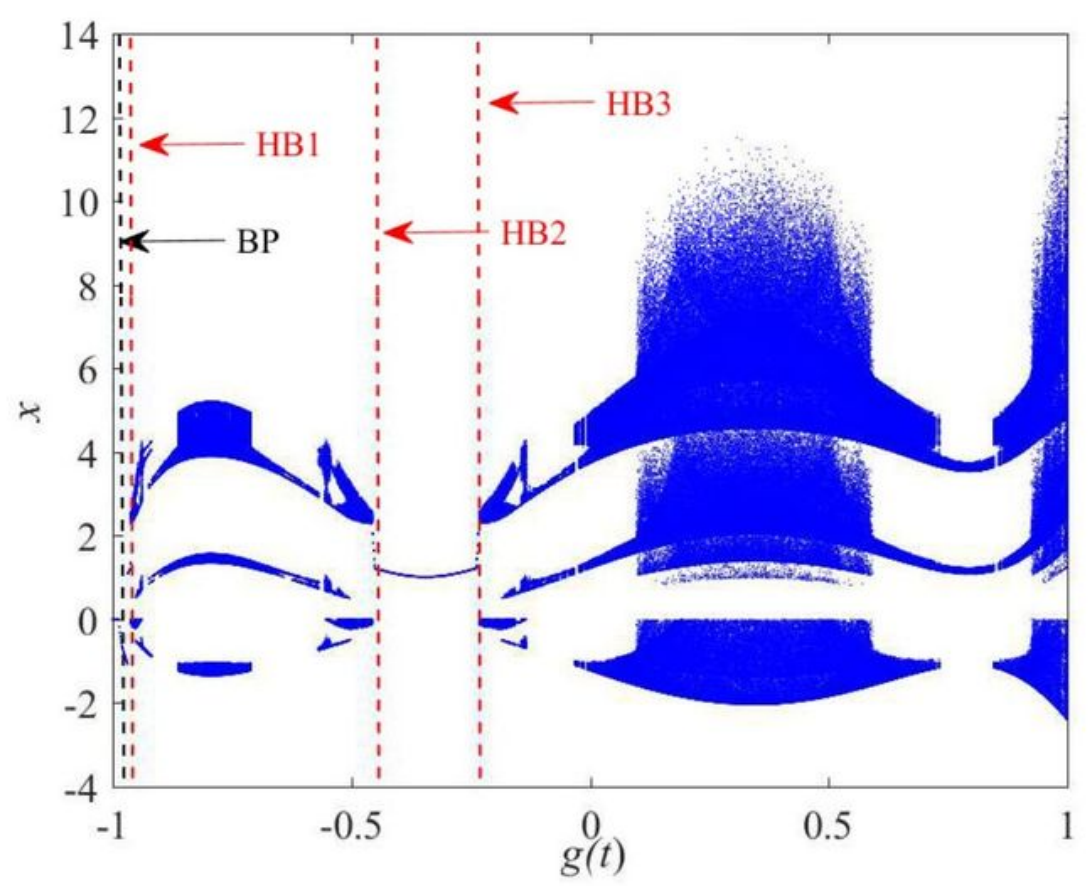

(a)

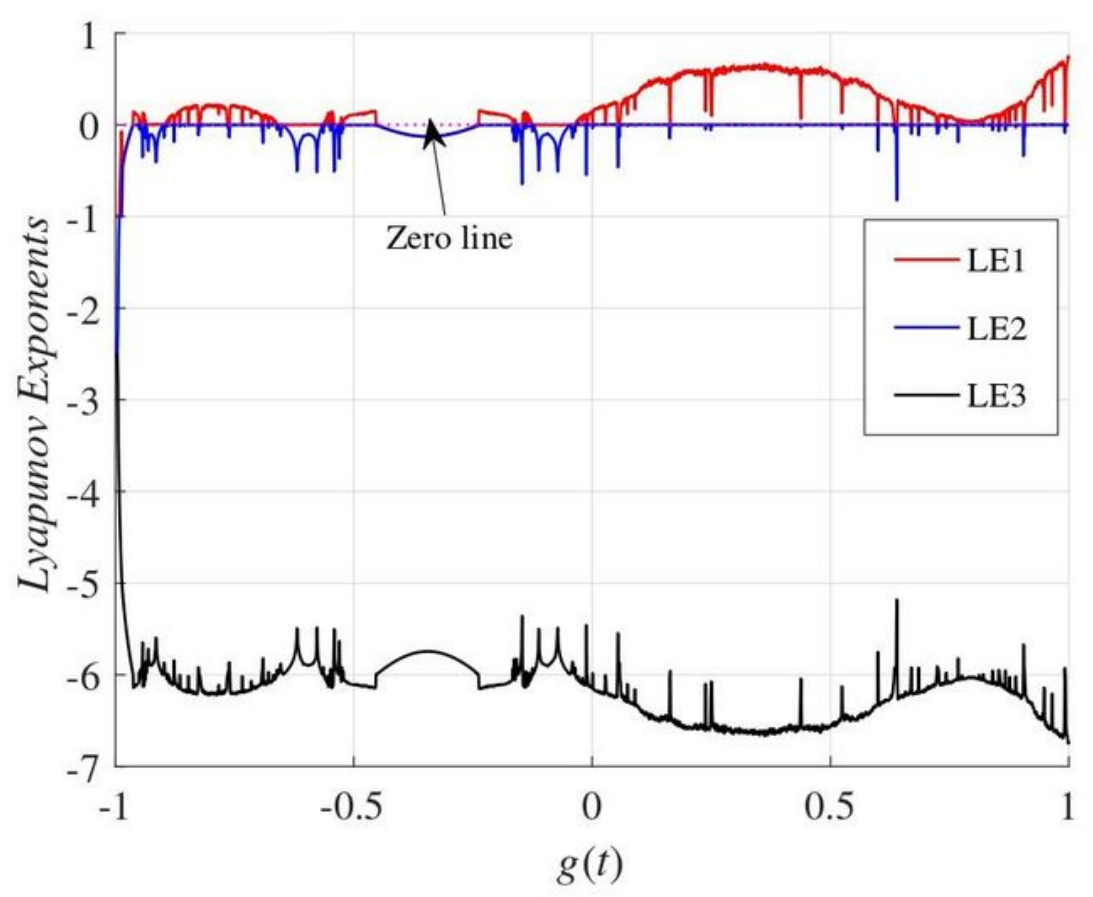

(b)

Figure 12

With $b=5, f 1=0.002$ and $f 2=0.01$, the dynamics of system (2) with $g(t)$ varying: (a) bifurcation diagram, (b) Lyapunov exponents. 


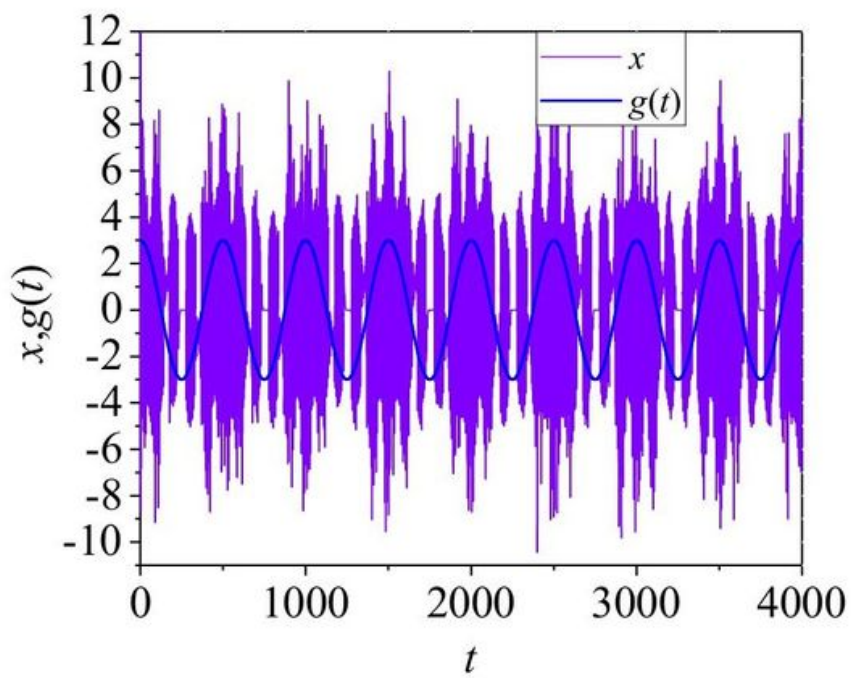

(a)

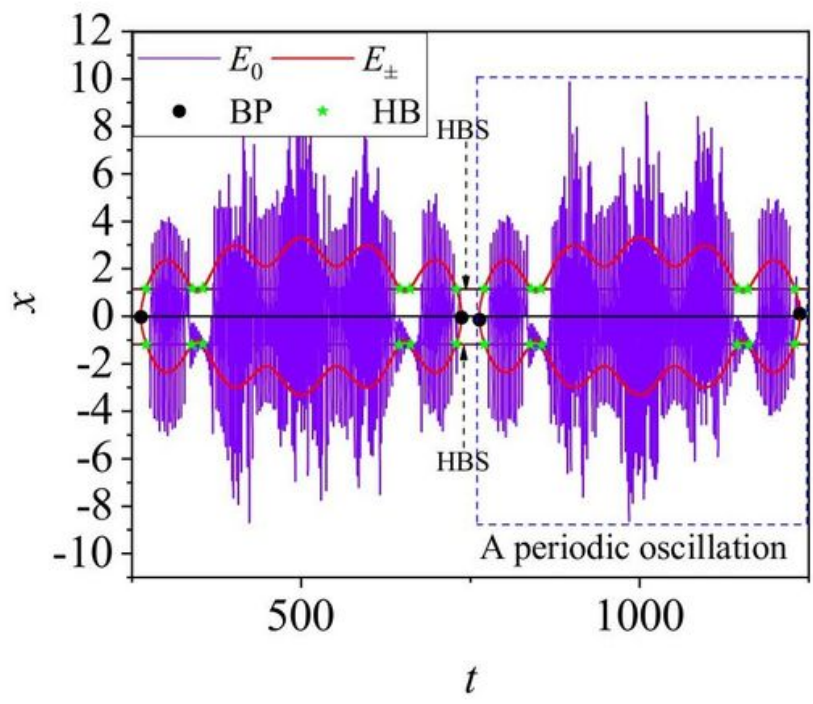

(c)

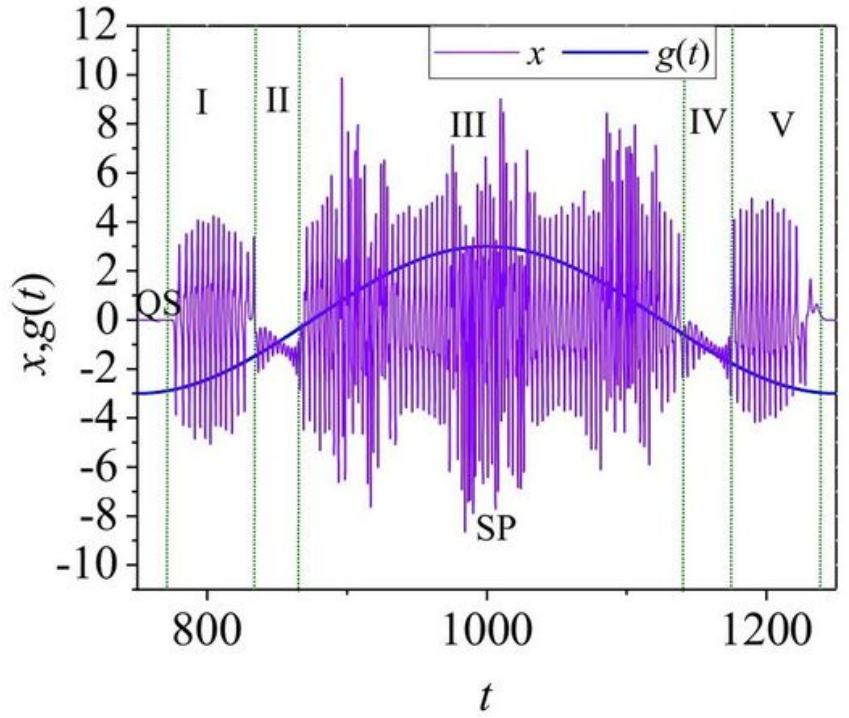

(b)

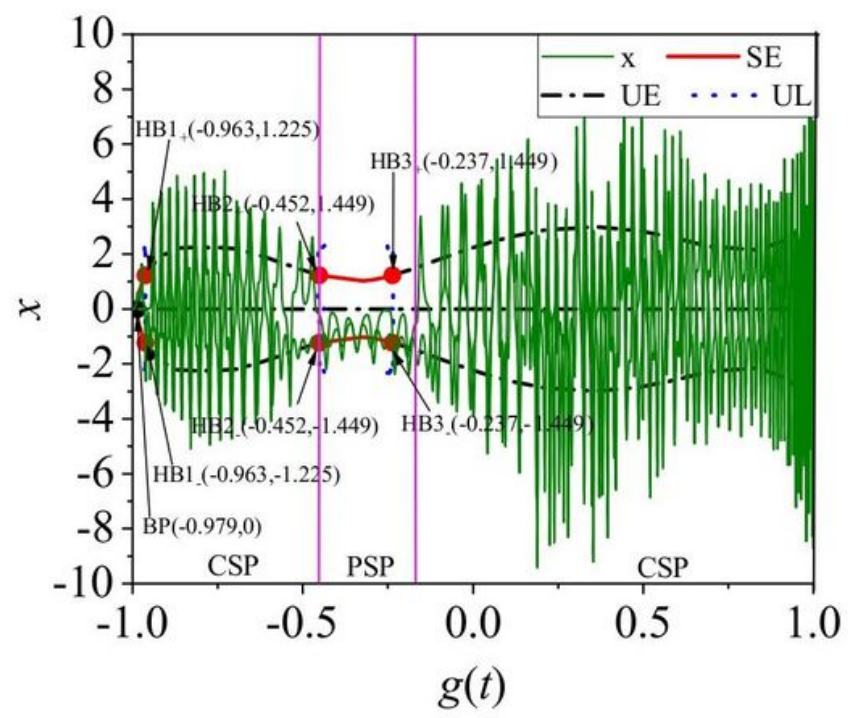

(d)

\section{Figure 13}

Fast-slow dynamic for $b=5$ : (a) whole time series of $x$ and $g(t)$, (b) Local enlarged time series of $x$ and $g(t),(c)$ The superposition graph for the evolution of equilibrium point and time series of $x$ along with time $t$, (d) transformed phase diagram. 


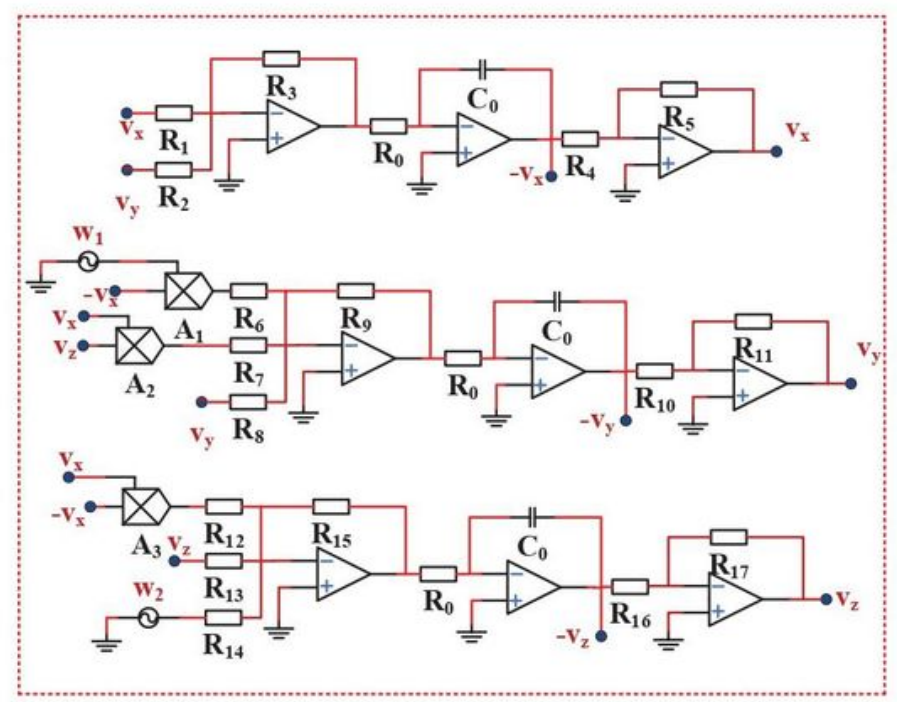

(a)

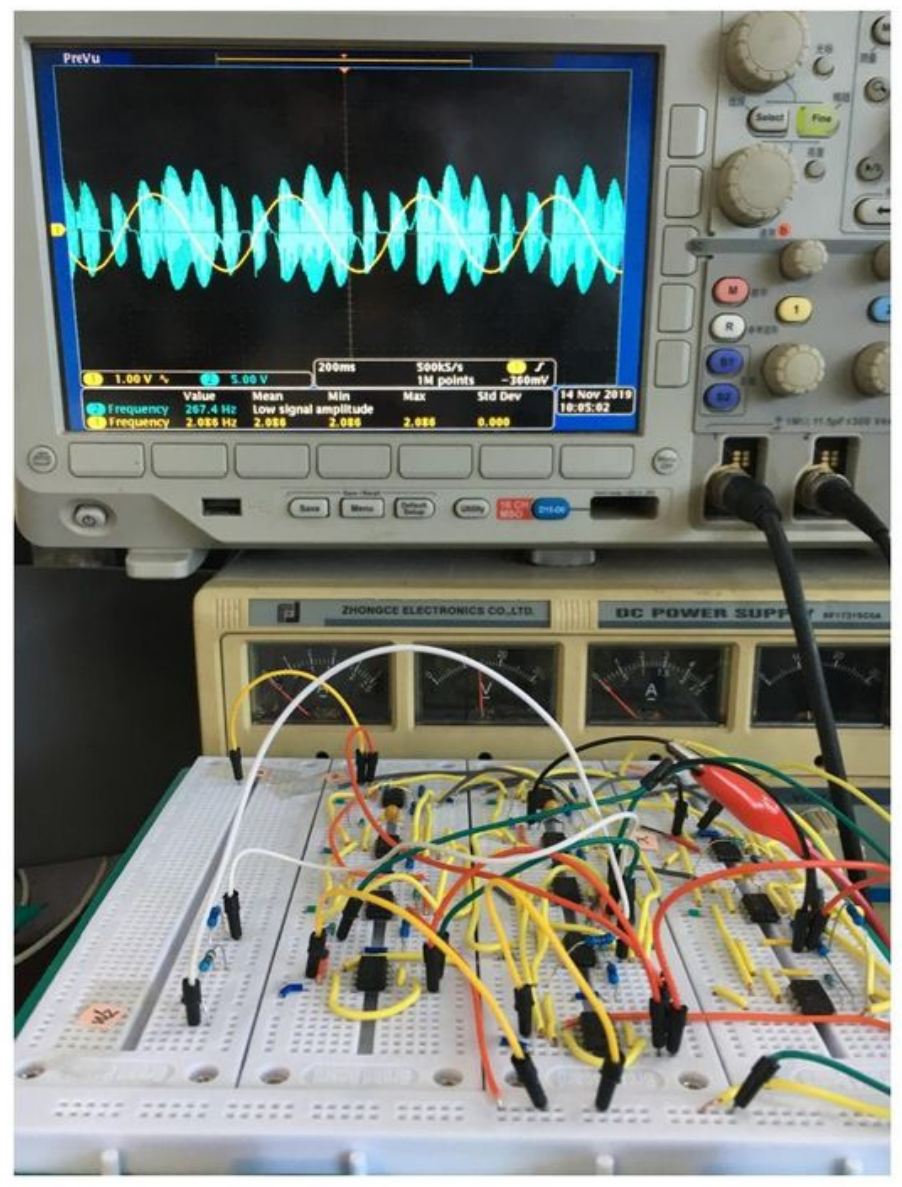

(b)

\section{Figure 14}

Circuit experimental verification: (a) the circuit schematic of system (1), (b) experimental setup: breadboard, power supply and os-cilloscope. 

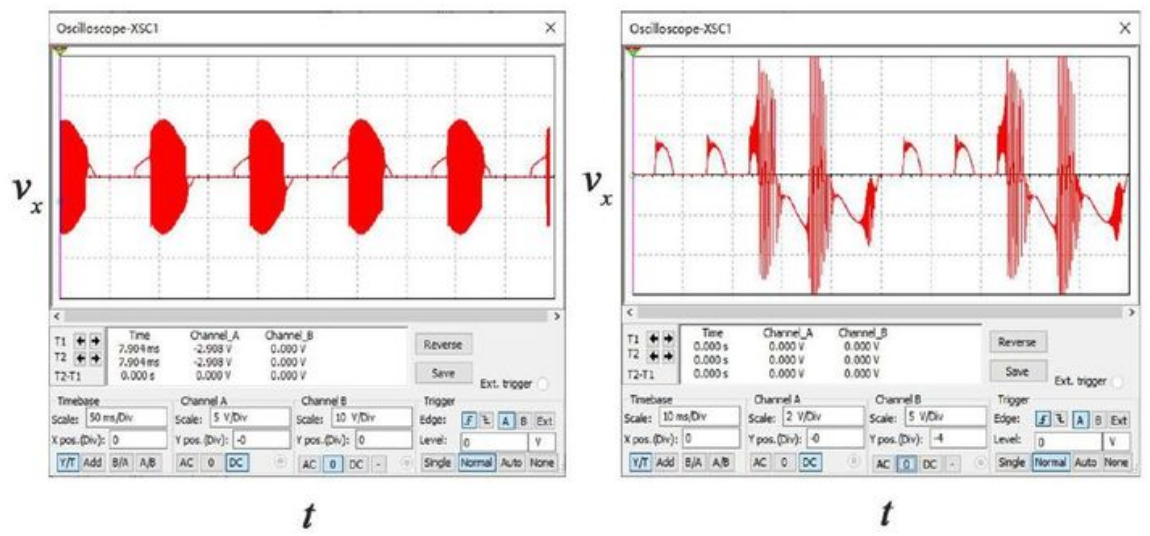

(a)

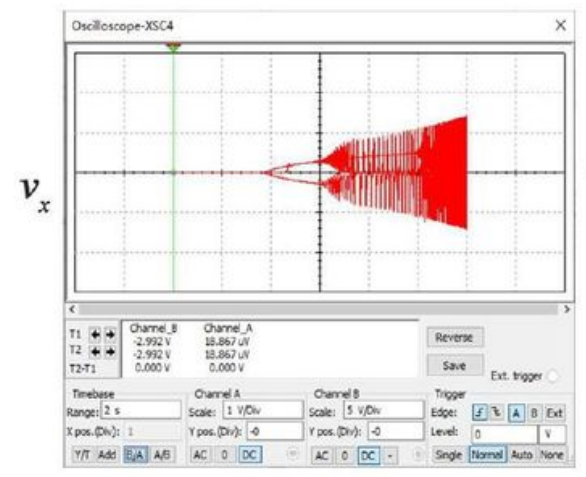

$g(t)$

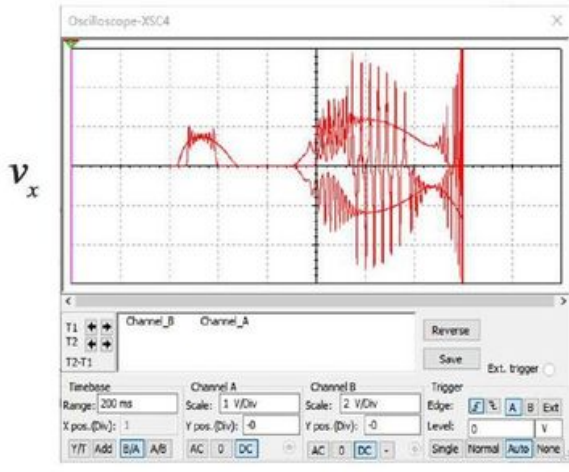

$g(t)$
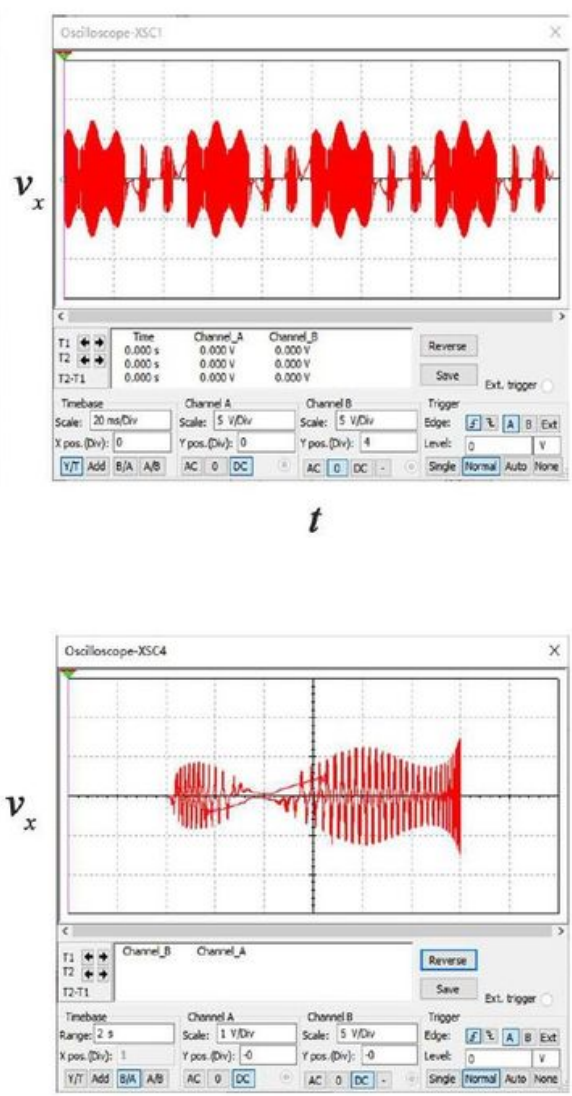

$g(t)$

(b)

\section{Figure 15}

With $\mathrm{R} 13=100 \mathrm{~K} \Omega$ and $\beta 1=\beta 2=3$, Multisim simulation results which time series and transformed phase diagram are shown for different parameters: (a) time series for case $1: f 1=f 2=10 \mathrm{~Hz}$ and $R 8=50 \mathrm{~K} \Omega$, case $2: \mathrm{f} 1=20 \mathrm{~Hz}, \mathrm{f} 2=100 \mathrm{~Hz}$ and $\mathrm{R} 8=50 \mathrm{~K} \Omega$, case $3: \mathrm{f} 1=20 \mathrm{~Hz}, \mathrm{f} 2=100 \mathrm{~Hz}, \mathrm{R} 8=25 \mathrm{~K} \Omega$, (b) transformed phase diagram for case $1: \mathrm{f} 1=\mathrm{f} 2=10 \mathrm{~Hz}$ and $\mathrm{R} 8=50 \mathrm{~K} \Omega$, case $2: \mathrm{f} 1=20 \mathrm{~Hz}, \mathrm{f} 2=100 \mathrm{~Hz}$ and $\mathrm{R} 8=50 \mathrm{~K} \Omega$, case $3: \mathrm{f} 1=20 \mathrm{~Hz}, \mathrm{f} 2=100 \mathrm{~Hz}, \mathrm{R} 8=25 \mathrm{~K} \Omega$. 


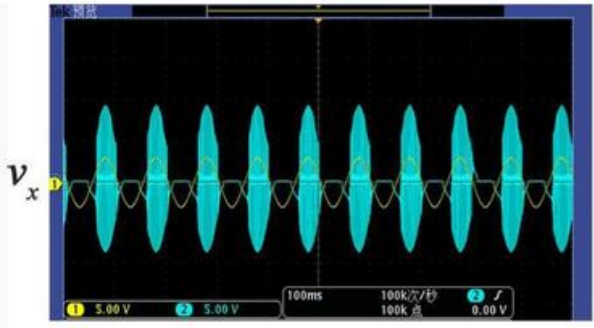

$t$

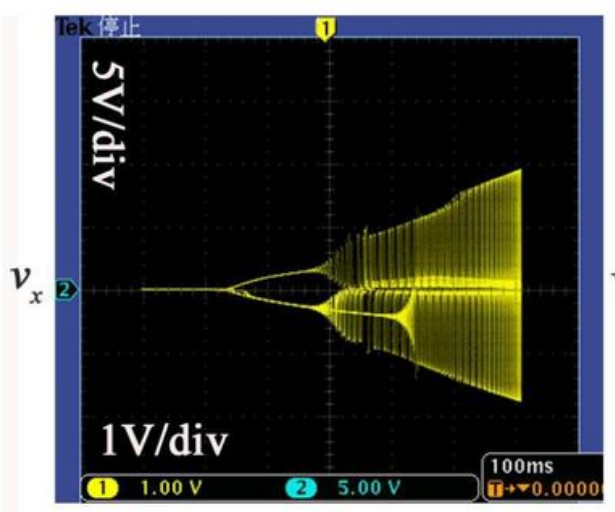

$g(t)$

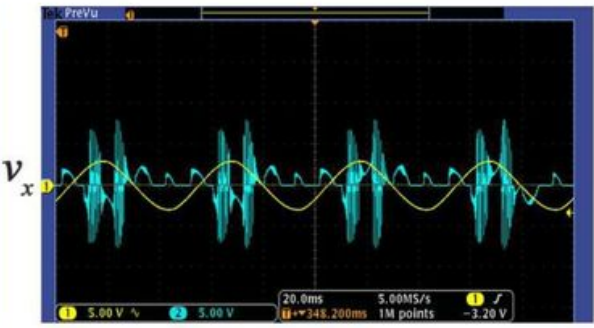

$t$

(a)

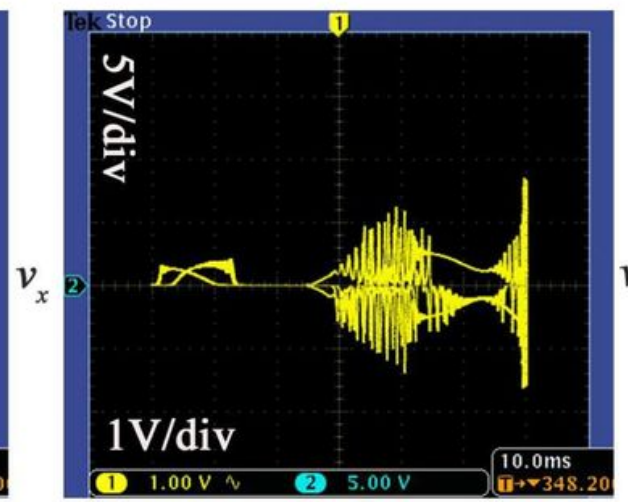

$g(t)$

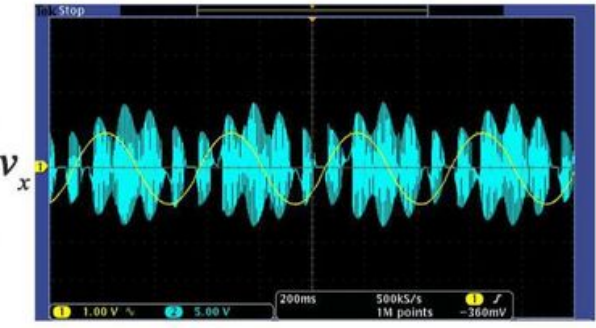

$t$

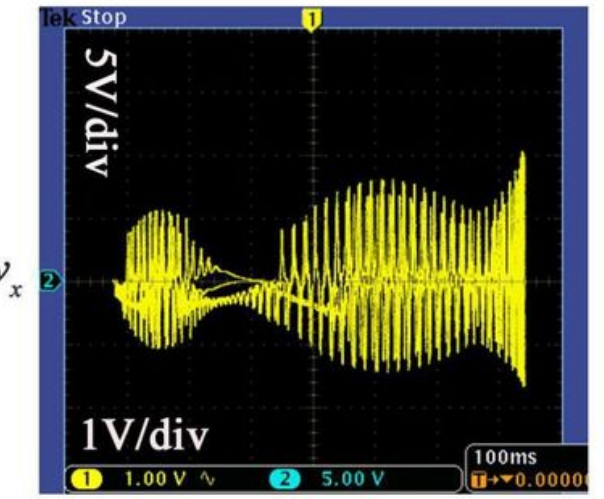

$g(t)$

(b)

\section{Figure 16}

6 Complex dynamics for varing-parameter are observed from oscilloscope (the detailed parameters correspond to Fig. 15): (a) time series of $x$, (b) transformed phase diagrams. 